\title{
THE DYNAMICS OF COSSERAT NETS
}

\author{
J. GRATUS AND R. W. TUCKER
}

Received 22 October 2001 and in revised form 10 July 2002

A formulation of the dynamics of a collection of connected simple 1dimensional Cosserat continua and rigid bodies is presented in terms of sections of an $\mathrm{SO}$ (3) fibration over a 1-dimensional net. A large class of junction conditions is considered in a unified framework. All the equations of motion and junction conditions are derived as extrema of a constrained variational principle on the net and are analysed perturbatively for structures with Kirchhoff constitutive properties. The whole discussion is based on the notion of a Cosserat net and its contractions obtained by taking certain limits that transform Cosserat elements to rigid structures. Generalisations are briefly discussed within this framework.

\section{Introduction}

Branched structures abound both in engineering and the natural world. A large class involves elastic members that can be approximated by interconnected 1-dimensional rods or strings. These include man-made assemblies such as bridges, oil rigs, antennae, and micromachines and biodynamic structures such as arterial pathways, trees, cobwebs, and macromolecules. In these, tube-like members may be directly connected together at nodes in space or to the boundaries of fixed or movable rigid bodies of arbitrary shape $[3,4,6,7,8,9,10,12,13,14,18,20,21]$. The elastodynamics of slender structures is a mature branch of mathematical physics [2] and is being increasingly recognised as a useful tool in Engineering and Biology. The simple Cosserat description of an isolated slender member offers a particularly concise and self-contained elastic 
model that can accommodate bending, shear, flexure, and torsion displacements of any size and can incorporate media of arbitrary constitutive complexity $[5,17]$. The equations of motion can be readily discretised and in many cases, where explicit details of cross-sectional stresses are not required, provide distinct computational advantages over more complex finite element modelling techniques.

In this paper, we are concerned with a general description of interconnected simple 1-dimensional Cosserat structures of arbitrary elastic type. These 1-dimensional Cosserat structures will be called Cosserat elements. The interconnections or junctions studied herein are one of the following types:

(i) the ends of two or more Cosserat elements are connected to each other at a single point. At this point, each element maintains a fixed angle relative to the others;

(ii) the ends of two or more Cosserat elements are connected to an idealised material point, which may possess mass, rotary inertia, or both. Again at this point, each element maintains a fixed angle relative to the others;

(iii) the ends of two or more Cosserat elements are connected to a single extended material rigid body. This means that the endpoint of an element is not necessarily coincident either with the centroid of the rigid body or any of the other elements connected to it. The ends of the elements maintain a fixed angle relative to any frame fixed within the rigid body.

It can be seen that the first case is a specialisation of the second which in turn is a specialisation of the third. The first case accommodates elements with sharp bends and those with sudden changes in their physical properties (density, stiffness, cross-section, etc.). In the same mathematical framework, we consider a single endpoint of a Cosserat element as either free or connected to a point mass or an extended rigid body. Although not considered in the paper, we can consider fixing this endpoint at a particular point in space by letting the mass of the point mass at the end of that Cosserat element tend to infinity. We can further fix the direction of the element at that point by letting the diagonal components of the moment of inertia tensor in the rigid body frame tend to infinity. By letting one or two diagonal components tend to infinity but not the others, we can constrain the endpoint of the element to rotate in certain directions. There are other possible junction conditions, some of which will be discussed in the conclusion.

The entire system of Cosserat elements, point masses, and extended rigid bodies in space will be called a Cosserat net. The equations of motion for a Cosserat net are second-order partial differential equations in two independent variables $t$ and $s$. In the standard Cosserat theory for a 


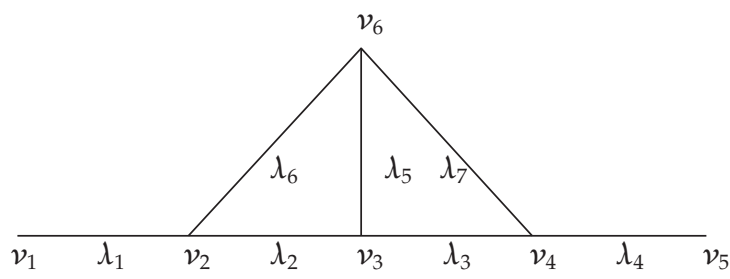

FIGURE 1.1. A net for a simple model of a suspension bridge, with 6 nodes labelled $v_{1}, \ldots, v_{6}$ and seven lines labelled $\lambda_{1}, \ldots, \lambda_{7}$.

single Cosserat element, the spatial variable $s$ typically runs from 0 to $L$, the stress-free length of the element. For Cosserat nets, the spatial variable $s$ labels points belonging to a topological space $\mathbf{Y}$ which we refer to as a net. To construct $\mathbf{Y}$, consider a set of compact intervals of the real line. Identify certain endpoints to create a junction, repeating this process to generate an arbitrary number of junctions. The net is connected if the resulting topological space is connected. The junctions and remaining endpoints are collectively called nodes and the intervals that connect these nodes are then termed lines. For an example, see Figure 1.1. Such a net is also called a 1-dimensional variety or a graph and here replaces the smooth "body manifold" for smooth 3-dimensional elastic bodies. In a more general setting, one could reformulate our definition of a net in the language of a "varifold" [1]. In a net, the independent variable $s$ labels both an internal point of any line as well as any node.

As well as the topology of the net $\mathbf{Y}$, the geometric data for the Cosserat net also contains a set of matrices and column vectors. For each node $v$ and line $\lambda$, which has an endpoint at $v$, there is a rotation matrix $\mathbf{X}(v, \lambda) \in \mathrm{SO}(3)$ and a column vector $\boldsymbol{\xi}^{d}(\nu, \lambda) \in \mathbb{R}^{3}$.

There are several dependent variables. These include the position in space of a point $s$ on an element at time $t$ given by $\underline{r}(t, s)$, the directors $\underline{d}_{1}(t, s), \underline{d}_{2}(t, s)$, and $\underline{d}_{3}(t, s)$ describing the internal state of the element, and a local angular velocity $\underline{w}(t, s)$. The directors form a frame field on $\mathrm{Y}$ and are generally chosen to be orthonormal with respect to the Euclidean metric on $\mathbb{R}^{3}$. In this paper, we do not assume that the tangent $r^{\prime}$ to the instantaneous space-curve $r(t, s)$ is always parallel to $\underline{d}_{3}$. Such dependent variables are all continuous in $s$ on each line but in general are discontinuous at each node. All such variables are assumed to be continuous and differentiable in $t$.

If $v$ is a node in the net and $\lambda$ is one of the lines connected to $v$, then we use the symbol $\underline{r}_{\lambda}(t, v)$ to be the position of the endpoint of the line $\lambda$ at node $v$. If $v$ includes a rigid body, the symbol $\underline{r}(t, v)$ (without the subscript) is the position of the centroid of the rigid body. (See Figure 1.2.) 


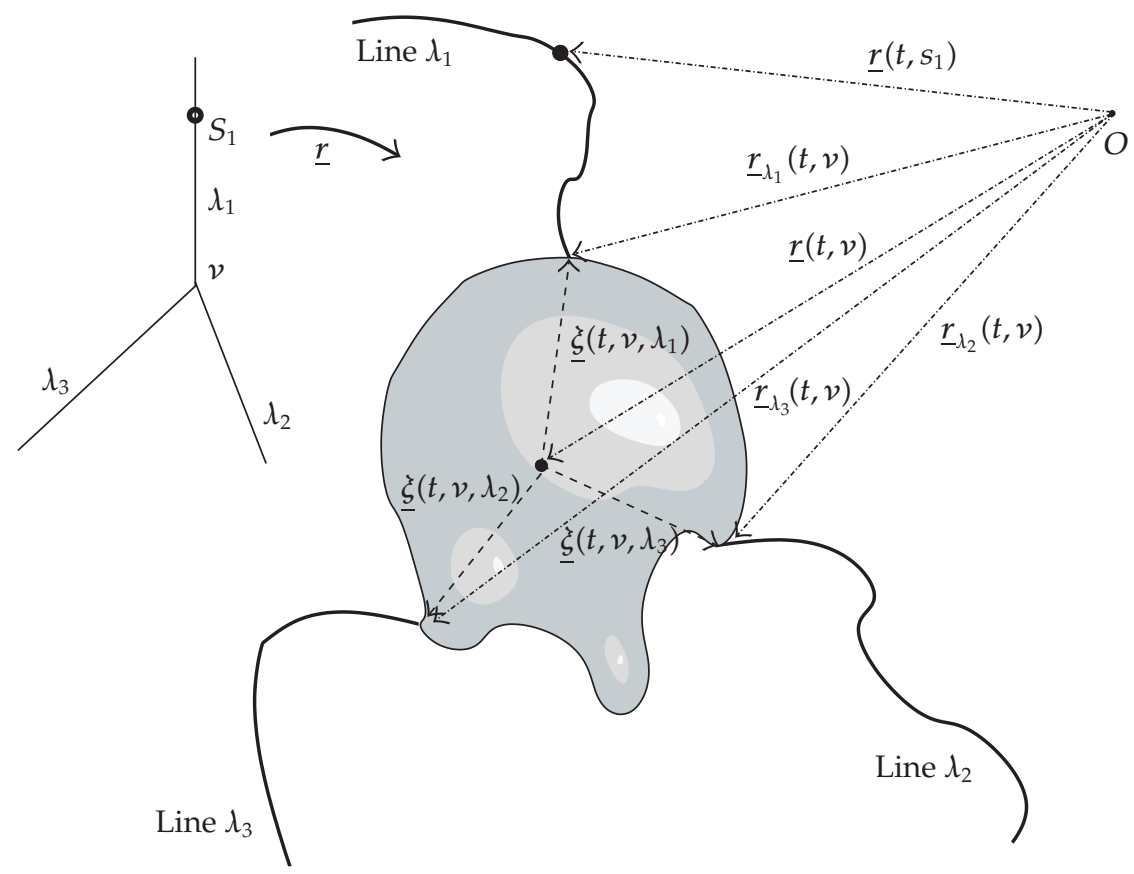

FIGURE 1.2. The map $\underline{r}(t, s)$ for an extended rigid junction. The node $v$ has three lines $\lambda_{1}, \lambda_{2}$, and $\lambda_{3}$ attached to it. The net on the left is mapped into the Cosserat net on the right.

If $v$ is a point junction, then $\underline{r}(t, v)=\underline{r}_{\lambda}(t, v)$ for all lines $\lambda$ which connect to $v$. If all the junctions are point junctions, then $\underline{r}(t, s)$ is a continuous function of $s$. If $v$ represents an extended junction, then there will be a difference between $\underline{r}(t, v)$ and $\underline{r}_{\lambda}(t, v)$ which is given by the vector $\underline{\xi}(t, v, \lambda)$ such that

$$
\underline{r}_{\lambda}(t, v)=\underline{r}(t, v)+\underline{\xi}(t, v, \lambda)
$$

where

$$
\underline{\xi}(t, v, \lambda)=\sum_{i=1}^{3} \xi_{i}^{d}(v, \lambda) \underline{d}_{i}(t, v)
$$

so the components $\boldsymbol{\xi}_{i}^{d}(\nu, \lambda)$ are independent of $t$.

The directors are in general discontinuous at the nodes. (See Figure 1.3.) We write $\left.\underline{d}_{j}\right|_{\lambda}(t, v)$ as the value of the $j$ th director at the endpoint of line $\lambda$ at $v$ (the vertical bar is used simply to distinguish subscripts). Furthermore, $\underline{d}_{j}(t, v)$ is the $j$ th member of a rigid body frame at $v$. If there is 


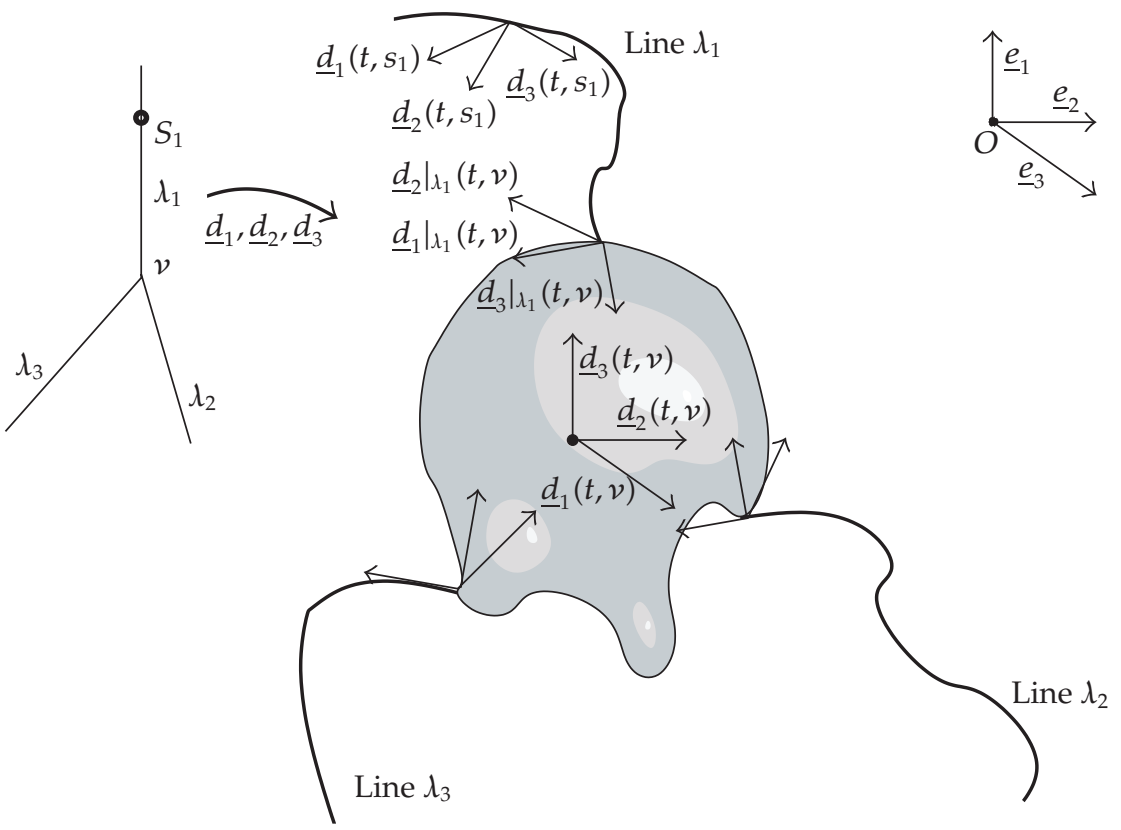

FIGURE 1.3. The images $\left[\underline{d}_{1}(t, s), \underline{d}_{2}(t, s), \underline{d}_{3}(t, s)\right]$ for an extended rigid junction. The node $v$ has three lines attached $\lambda_{1}, \lambda_{2}$, and $\lambda_{3}$ and $\left[\underline{d}_{1}(t, v), \underline{d}_{2}(t, v), \underline{d}_{3}(t, v)\right]$ is a frame for the rigid body attached at its centroid. The director frame $\left[\left.\underline{d}_{1}\right|_{\lambda_{1}}(t, v),\left.\underline{d}_{2}\right|_{\lambda_{1}}(t, v),\left.\underline{d}_{3}\right|_{\lambda_{1}}(t, v)\right]$ is the limit at the contact point of the frame along the element labelled by line $\lambda_{1}$. The vectors $\left[\underline{e}_{1}, \underline{e}_{2}, \underline{e}_{3}\right]$ constitute a fixed global inertial reference frame in space.

no rigid body at $v$, it is still necessary to define the frame $\underline{d}_{1}(t, v), \underline{d}_{2}(t, v)$, and $\underline{d}_{3}(t, v)$, which can be done by setting $\underline{d}_{j}(t, v)=\left.\underline{d}_{j}\right|_{\lambda}(t, v)$, where $\lambda$ has an endpoint at $v$. The discontinuities in the frame are defined by

$$
\sum_{k=1}^{3} \mathbf{X}_{k j}(v, \lambda) \underline{d}_{k}(t, v)=\left.\underline{d}_{j}\right|_{\lambda}(t, v),
$$

where $\mathbf{X}(\nu, \lambda)$ is the time independent rotation matrix introduced above. This constraint comes from the statement that the Cosserat elements maintain a fixed angle relative to the frame of the rigid body or point mass. In Section 2.3, it is shown how this condition can be reformulated in terms of an associated rotation matrix $\mathbf{A}(t, s)$ that is continuous in both $s$ and $t$ on $\mathbb{R} \times \mathbf{Y}$.

The position $\underline{r}$ and frame $\left\{\underline{d}_{j}\right\}$ associated with an interior point on a line $\lambda$ within the net at time $t$ are given by the Cosserat equations of 
motion. In nondimensional form, these are given by

$$
\begin{gathered}
\mu \underline{\ddot{r}}=\underline{n}^{\prime}+\underline{f}, \\
(\underline{\mathbf{I}}(\underline{w}))^{\cdot}=\underline{m}^{\prime}+\kappa \underline{r}^{\prime} \times \underline{n}+\underline{l},
\end{gathered}
$$

where $\mu$ and $\underline{I}$ are the rescaled mass and rotary inertia variables of $\lambda$ and $f, \underline{l}$ denote the external force and torque, $\underline{n}$ and $\underline{m}$ denote the contact force and torque which are given by material constitutive relations, and $\kappa$ is a dimensionless rescaling parameter. The dot and prime denote differentiation with respect to $t$ and $s$, respectively. The equations describing the position and orientation of an idealised massive point particle with rotary inertia at node $v$ and time $t$ are given by

$$
\begin{gathered}
M_{\mathcal{v}} \underline{\ddot{r}}(t, v)=\underline{F}(t, v)-\sum_{\lambda} \Omega(v, \lambda) \underline{n}_{\lambda}(t, v), \\
\left(\underline{\mathbf{I}}_{v} \underline{w}(t, v)\right)^{\cdot}=\underline{L}(t, v)-\sum_{\lambda} \Omega(v, \lambda) \underline{m}_{\lambda}(t, v),
\end{gathered}
$$

where the sum is over all lines $\lambda$ terminating at $v$.

Here, $M_{v}, \underline{\mathbf{I}}_{v}, \underline{F}(t, v)$, and $\underline{L}(t, v)$ are, respectively, the mass, moment of inertia, external force, and torque applied to node $v$, and $\underline{n}_{\lambda}(t, v)$ and $\underline{m}_{\lambda}(t, v)$ are the limit of the contact force and torque at node $v$ along the line $\lambda$. The symbol $\Omega(\nu, \lambda)= \pm 1$ depends on an arbitrarily specified orientation assigned to line $\lambda$. (With the convention $\Omega(\nu, \lambda)=0$ if neither end of line $\lambda$ is at node $\nu$, the sums above can be extended to all lines in the net). If $v$ is an extended rigid junction, then (1.6) is generalised to

$$
\left(\underline{\mathbf{I}}_{v} \underline{w}(t, v)\right)^{\cdot}=\underline{L}(t, v)-\sum_{\lambda} \Omega(v, \lambda)\left(\underline{m}_{\lambda}(t, v)+\kappa \underline{\xi}(t, v, \lambda) \times \underline{n}_{\lambda}(t, v)\right) .
$$

In the case that the number of lines at the junction $v$ is one so that $v$ is the endpoint of some line $\lambda$, then the junction condition (1.1) and (1.3) and the equations of motions of the junction (1.5) and (1.7) reduce to the case of a single Cosserat element attached to a massive extended body. Such structures were studied in some detail by Simo et al. [16].

In Section 2, we give the mathematical background for this paper. This includes the divergence theorem and Euler-Lagrange equations for a net, a discussion of reference configurations, and the introduction of the matrix A mentioned above.

Variational derivations of the equations of motion for elastic structures and especially Cosserat elements are well established, see, for example, [15] and references therein. Section 3 follows the Lagrangian approach to derive the equations of motion for a Cosserat net with point junction conditions (1.5), (1.6) and hyperelastic elements. This serves 
largely to establish the variational notation and tools for the more intricate derivation of the equations of motion for hyperelastic nets that include extended junctions carried out in Section 4 . The variational method relies on maintaining geometric constraints during the variation and the extensive use of a global field of $\mathrm{SO}(3)$ matrices and their derivatives on the net. The efficacy of using such group elements in variational calculations has been stressed by a number of authors [15, 19]. We believe that their implementation in the context of Cosserat nets is new for both the computation of variational derivatives and the linearisation of the equations of motion about static stress-free configurations. After expressing equations in nondimensional form in Section 5, this linearisation is carried out in Section 6 where the junction conditions are analysed in order to reduce the system to an eigenproblem for normal modes.

Although our variational formulation is ideally suited to an analysis of the nonlinear stability [19] of a Cosserat net, we do not pursue this here since the choice of appropriate norms is beyond the scope of this article. However, the linear stability analysis about stress-free configurations offers valuable information that can be exploited in numerical modelling schemes where discretisation of the partial differential equations of the net can be discretised in a basis of normal modes. Indeed, practical applications of our linearised analysis has led to the development of the Maple computer program LINCOSS [11] which can be used to estimate the mode spectrum and associated eigenmodes of (in principle arbitrary) Cosserat nets containing rigid extended structures.

Finally, in Section 7, we show that by making the stiffness of any Cosserat element in a net tend to infinity, one can generate a new net where such an element is replaced by an extended rigid body node.

\section{Mathematical background}

\subsection{The structure of nets}

As mentioned in the introduction, the net $\mathbf{Y}$ consists of a set of nodes which will be written as $\mathcal{N}(\mathbf{Y})=\left\{v_{1}, v_{2}, \ldots, v_{N_{\text {nodes }}}\right\}$. These are connected via a set of lines, written as $\mathcal{L}(\mathbf{Y})=\left\{\lambda_{1}, \lambda_{2}, \ldots, \lambda_{N_{\text {lines }}}\right\}$. To simplify notation, two types of net are not discussed: the first is a single loop with no nodes on it and the second is any net containing a loop with a single node on it.

The space $\mathbf{Y}$ is given an orientation. Thus for each line $\lambda \in \mathcal{L}(\mathbf{Y})$, one associates a direction. This choice of direction is arbitrary. A coordinate system on $\mathbf{Y}$ is a bijective map from each line onto a closed interval of the real line such that the orientation arrow for each line indicates the direction of an increasing coordinate. The symbol $s_{\mathcal{\lambda}}$ is used to represent the coordinate on the line $\lambda$. 
The boundary of $\mathbf{Y}$ is given by the set of ordered pairs $\partial \mathbf{Y}=\{(v, \lambda) \in \mathcal{N}(\mathbf{Y}) \times \mathcal{L}(\mathbf{Y}) \mid$ node $v$ is at one of the ends of line $\lambda\}$.

Thus the number of ordered pairs in the set $\partial \mathbf{Y}$ is twice the number of lines. For example, the boundary of the "bridge" given in Figure 1.1 consists of the ordered pairs

$$
\begin{aligned}
\partial \mathbf{Y}=\left\{\left(v_{1}, \lambda_{1}\right),\left(v_{2}, \lambda_{1}\right),\left(v_{2}, \lambda_{2}\right),\left(v_{2}, \lambda_{6}\right),\left(v_{3}, \lambda_{2}\right),\left(v_{3}, \lambda_{3}\right),\left(v_{3}, \lambda_{5}\right),\right. \\
\\
\left.\left(v_{4}, \lambda_{3}\right),\left(v_{4}, \lambda_{4}\right),\left(v_{4}, \lambda_{7}\right),\left(v_{5}, \lambda_{4}\right),\left(v_{6}, \lambda_{5}\right),\left(v_{6}, \lambda_{6}\right),\left(v_{6}, \lambda_{7}\right)\right\} .
\end{aligned}
$$

The boundary inherits an orientation from $\mathbf{Y}$. This is the map

$$
\begin{aligned}
& \Omega: \mathcal{N}(\mathbf{Y}) \times \mathcal{L}(\mathbf{Y}) \longmapsto\{-1,0,+1\} \\
& \Omega(v, \lambda)= \begin{cases}+1 & \text { if }(\nu, \lambda) \in \partial \mathbf{Y} \text { and arrow on } \lambda \text { points towards } v \\
-1 & \text { if }(\nu, \lambda) \in \partial \mathbf{Y} \text { and arrow on } \lambda \text { points away from } v, \\
0 & \text { if }(v, \lambda) \notin \partial \mathbf{Y} .\end{cases}
\end{aligned}
$$

Given a function $f: \mathbf{Y} \mapsto \mathbb{R}$, we define a set of restricted functions

$$
f_{\lambda}=\left.f\right|_{\text {line } \lambda} \text {. }
$$

If each $f_{\lambda}$ is continuous, then $f$ is said to be line continuous. In this case, the limit

$$
f_{\lambda}(v)=\lim _{s \rightarrow v} f_{\lambda}(s)
$$

exists. For $f$ to be continuous, requires that $f$ is line continuous and that

$$
f(v)=f_{\lambda}(v)
$$

is independent of $\lambda$. If $f$ is not continuous at $v$, it is useful in some cases to define $f(v)$ which, in general, will not satisfy (2.6).

We can differentiate $f$ to give $f^{\prime}$, where

$$
\left.f^{\prime}\right|_{\text {line } \curlywedge}=\left(f_{\lambda}\right)^{\prime} .
$$

We note that, in general, if $f$ is continuous, then $f^{\prime}$ need only to be line continuous. The one form $d f$ is given by $\left.d f\right|_{\text {line } \lambda}=f_{\lambda}^{\prime} d s_{\lambda}$. This is only defined in the interior of $\mathbf{Y}$ (i.e., excluding the nodes). 
As mentioned in the introduction, there are two independent variables in the dynamics of Cosserat nets. As above, differentiation with respect to $s$ will be denoted with a prime, and differentiation with respect to $t$ by a dot. Thus $\partial \underline{r} / \partial s=\underline{r}^{\prime}, \partial \underline{r} / \partial t=\underline{\dot{r}}$, and so forth.

In the Cosserat theory of nets, presented here, all the dependent variables $\underline{r}(t, s), \mathbf{A}(t, s), \underline{w}(t, s), \underline{n}(t, s)$, and $\underline{m}(t, s)$, etc. are line continuous in their spatial variable $s$, and continuous in the temporal variable $t$. Furthermore, certain dependent variables $\mathbf{A}(t, s)$ and $\underline{w}(t, s)$ are continuous in both $s$ and $t$ on $\mathbf{Y}$.

The above formalism readily extends the divergence theorem on manifolds to 1-dimensional varieties.

LEMMA 2.1. Let $f: \mathbf{Y} \mapsto \mathbb{R}$ be line continuous. Then

$$
\int_{\mathbf{Y}} d f=\int_{\partial \mathbf{Y}} f
$$

where

$$
\int_{\mathbf{Y}} d f=\sum_{\lambda \in \mathcal{L}(\mathbf{Y})} \int_{\text {line } \lambda} d f_{\lambda,}, \quad \int_{\partial \mathbf{Y}} f=\sum_{(\nu, \lambda) \in \partial \mathbf{Y}} f_{\lambda}(\mathcal{v}) \Omega(v, \lambda) .
$$

Proof. If line $\lambda$ connects $v_{0}$ and $v_{1}$ with the arrow pointing from $v_{0}$ to $v_{1}$, then

$$
\int_{\text {line } \lambda} d f_{\lambda}=\int_{\text {line } \lambda} \frac{d f_{\lambda}}{d s_{\lambda}} d s_{\lambda}=f_{\lambda}\left(v_{1}\right)-f_{\lambda}\left(v_{0}\right)=\sum_{v \in \mathcal{N}(\mathbf{Y})} \Omega(v, \lambda) f_{\lambda}(v)
$$

Since $\Omega\left(v_{0}, \lambda\right)=-1, \Omega\left(v_{1}, \lambda\right)=1$, and $\Omega\left(v_{0}, \lambda\right)=0$ otherwise. Summing over all lines gives

$$
\sum_{\lambda \in \mathcal{L}(\mathbf{Y})} \int_{\text {line } \mathcal{\lambda}} d f_{\lambda}=\sum_{\lambda \in \mathcal{L}(\mathbf{Y})} \sum_{\mathcal{v} \in \mathcal{N}(\mathbf{Y})} \Omega(\mathcal{v}, \lambda) f_{\lambda}(\mathcal{v}) .
$$

However, since $\Omega(\nu, \lambda)=0$ if $(\nu, \lambda) \notin \partial Y$, then we can change the sum on the left-hand side to complete the proof.

\section{Euler-Lagrange equations for varieties}

In section 3, we derive the Cosserat net equations from a single action. Although this is a standard application of the variational principle with Lagrange multipliers to handle the constraints, the proof is quite long. In order to show the origin of junction conditions from an action principle on varieties, we execute the process here for a simpler Lagrangian. 
Consider the following action functional on a general 1-dimensional variety $\mathbf{Y}$ :

$$
\mathcal{S}=\mathcal{S}\left(x, x^{\prime}\right)=\int_{\mathbf{Y}} \mathcal{L}\left(s, x, x^{\prime}\right) d s+\sum_{\nu \in \mathcal{N}(\mathbf{Y})} \mathcal{L}^{\text {node }}(\nu, x),
$$

where $\mathcal{L}: \mathbf{Y} \times \mathbb{R} \times \mathbb{R} \mapsto \mathbb{R}$ is line continuous, $\mathcal{L}^{\text {node }}: \mathcal{N}(\mathbf{Y}) \times \mathbb{R} \mapsto \mathbb{R}$ is continuous, and $x: \mathbf{Y} \mapsto \mathbb{R}$ is continuous. The fact that $\mathcal{L}$ is not defined at $\mathcal{v}$ is not important since it does not affect the integration.

Varying $\mathcal{S}$ with respect to $x$ and $x^{\prime}$ gives

$$
\delta \mathcal{S}=\int_{\mathbf{Y}}\left(\frac{\partial \mathcal{L}}{\partial x} \delta x+\frac{\partial \mathcal{L}}{\partial x^{\prime}} \delta x^{\prime}\right) d s+\sum_{v \in \mathcal{N}(\mathbf{Y})} \frac{\partial \mathcal{L}^{\text {node }}}{\partial x} \delta x
$$

Integrating by parts and using (2.8) gives

$$
\delta \mathcal{S}=\int_{\mathbf{Y}}\left(\frac{\partial \mathcal{L}}{\partial x}-\frac{d}{d s} \frac{\partial \mathcal{L}}{\partial x^{\prime}}\right) \delta x d s+\int_{\partial \mathbf{Y}} \frac{\partial \mathcal{L}}{\partial x^{\prime}} \delta x+\sum_{\nu \in \mathcal{N}(\mathbf{Y})} \frac{\partial \mathcal{L}^{\text {node }}}{\partial x} \delta x
$$

Letting $\delta x$ have support only on the interior of $\mathbf{Y}$ gives the standard Euler-Lagrange equations. By writing the integral $\int_{\partial \mathrm{Y}}$ using (2.9), the natural junction conditions at $v \in \mathcal{N}(\mathbf{Y})$ follow as

$$
\frac{\partial \mathcal{L}^{\text {node }}}{\partial x}+\sum_{\lambda}\left(\frac{\partial \mathcal{L}}{\partial x^{\prime}}\right)_{\lambda}(\mathcal{v}) \Omega(\nu, \lambda)=0
$$

The sum $\sum_{\lambda}$ may be considered to be over all $\lambda$ or just those which have an end at node $v$. These are equivalent since $\Omega(v, \lambda)=0$ otherwise. The expression $\left(\partial \mathcal{L} / \partial x^{\prime}\right)_{\mathcal{\lambda}}(\mathcal{V})$ means evaluate $\partial \mathcal{L} / \partial x^{\prime}$ as a function on $\mathbf{Y}$ and then take its limit as $s \rightarrow \mathcal{v}$ along line $\lambda$.

\subsection{Vectors, frames, and matrices}

In this section, essential notational constructions are presented. One has to be careful in distinguishing between the manifold $\mathcal{M}=\mathbb{R}^{3}$, which is the physical space in which the motion of $\mathbf{Y}$ takes place, and the space $\mathcal{C}=\mathbb{R}^{3}$ of column- 3 vectors with entries which represent the components of the vectors with respect to the different orthonormal bases employed.

Throughout this paper, the following conventions are used:

(i) vectors (belonging to $\mathcal{M}$ or $T \mathcal{M}$ ) are written as unbold lowercase letters with an underscore, for example, $\underline{r}$ and $\underline{w}$. Since $\mathcal{M}$ is flat, and $T_{\underline{x}} \mathcal{M} \cong \mathcal{M}$ for any point $\underline{x} \in \mathcal{M}$; 
(ii) $\left(\begin{array}{l}1 \\ 1\end{array}\right)$ tensors on $\mathcal{M}$ are written as bold capital letters with an underscore, for example, $\underline{\mathbf{I}}$ and $\mathbf{J}$;

(iii) column-3 vectors are written as bold lowercase letters, for example, $\mathbf{x}$ and $\mathbf{y}$;

(iv) $3 \times 3$ matrices are written as bold capital letters, for example, $\mathbf{A}$ and $\mathbf{D}$;

(v) matrices and column vectors with an $e$ superscript, for example, $\mathbf{T}^{e}$ and $\mathbf{x}^{e}$, are the components of the corresponding tensors and vectors ( $\underline{\mathbf{T}}$ and $\underline{x}$ ) in the fixed frame $\underline{e}_{1}, \underline{e}_{2}, \underline{e}_{3}$. See below;

(vi) matrices and column vectors with a $d$ superscript, for example, $\mathbf{T}^{d}$ and $\mathbf{x}^{d}$, are the components of the corresponding tensors and vectors $\left(\mathbf{T}^{d}\right.$ and $\mathbf{x}^{d}$ ) in the local director frame $\underline{d}_{1}, \underline{d}_{2}, \underline{d}_{3}$. See below;

(vii) in Section 5, objects with inverted hats $\left(^{\circ}\right)$ on carry dimensions based on mass, time, and length;

(viii) in Section 6, objects with hats $(\stackrel{*}{)}$ ) or tildes $(\cdot)$ are perturbations;

(ix) in Section 7 , objects with tildes ( $\left(^{*}\right)$ will be explained there.

There are two preferred orthonormal frame fields on $\mathcal{M}$. The first is a fixed (global Euclidean-parallel) basis $\left\{\underline{e}_{1}, \underline{e}_{2}, \underline{e}_{3}\right\}$. The other is called a local director basis. Restricted to $Y$, it is written $\left\{\underline{d}_{1}, \underline{d}_{2}, \underline{d}_{3}\right\}$, depends on both time $t$ and $s$, and is identified with a Cosserat director frame for elements in the net. At each point in space and at time $t$, these two bases are related by a local rotation tensor $\underline{\mathbf{D}}(t, s)$,

$$
\underline{d}_{i}=\underline{\mathbf{D}}\left(\underline{e}_{i}\right), \quad \underline{e}_{i}=\underline{\mathbf{D}}^{-1}\left(\underline{d}_{i}\right) .
$$

The components of a general vector $\underline{x} \in \mathcal{M}$, with respect to the fixed and director basis, are written as column vectors

$$
\mathbf{x}^{e}=\left(\begin{array}{l}
\underline{x} \cdot \underline{e}_{1} \\
\underline{x} \cdot \underline{\underline{e}}_{2} \\
\underline{x} \cdot \underline{\underline{e}}_{3}
\end{array}\right)=\underline{x} \cdot \underline{e}_{i} \mathbf{e}_{i}, \quad \mathbf{x}^{d}=\left(\begin{array}{l}
\underline{x} \cdot \underline{d}_{1} \\
\underline{\underline{x}} \cdot \underline{d}_{2} \\
\underline{\underline{d}}
\end{array}\right)=\underline{x} \cdot \underline{d}_{3} \underline{\mathbf{d}}_{i} .
$$

The superscript $e$ and $d$ are used to distinguish between the two frames. Here, $\left\{\mathbf{e}_{i}\right\}$ is the natural basis of $\mathcal{C}=\mathbb{R}^{3}$ given by

$$
\mathbf{e}_{1}=\left(\begin{array}{l}
1 \\
0 \\
0
\end{array}\right), \quad \mathbf{e}_{2}=\left(\begin{array}{l}
0 \\
1 \\
0
\end{array}\right), \quad \mathbf{e}_{3}=\left(\begin{array}{l}
0 \\
0 \\
1
\end{array}\right)
$$


Thus $\mathbf{x}^{e}$ and $\boldsymbol{x}^{d}$ are related by the matrix $\mathbf{D} \in \mathrm{SO}(3)$ with entries $\mathbf{D}_{i j}=$ $\underline{e}_{i} \cdot \underline{d}_{j}$

$$
\mathbf{x}^{e}=\mathbf{D} \mathbf{x}^{d}, \quad \mathbf{x}^{d}=\mathbf{D}^{T} \mathbf{x}^{e} .
$$

In $\mathcal{M},\left(\begin{array}{l}1 \\ 1\end{array}\right)$ tensors, in these bases, become $3 \times 3$ matrices with entries

$$
\mathbf{T}_{i j}^{e}=\underline{e}_{i} \cdot \underline{\mathbf{T}}\left(\underline{e}_{j}\right), \quad \mathbf{T}_{i j}^{d}=\underline{d}_{i} \cdot \underline{\mathbf{T}}\left(\underline{d}_{j}\right) .
$$

Thus

$$
\mathbf{T}^{e}=\mathbf{D T}^{d} \mathbf{D}^{T}, \quad \mathbf{T}^{d}=\mathbf{D}^{T} \mathbf{T}^{e} \mathbf{D} .
$$

The Lie algebra so(3) of antisymmetric $3 \times 3$ matrices is isomorphic to $\mathcal{C}=\mathbb{R}^{3}$ as a vector space and given by the map

$$
\Lambda: \mathcal{C} \longmapsto \operatorname{so}(3) \quad \Lambda\left(\begin{array}{l}
x_{1} \\
x_{2} \\
x_{3}
\end{array}\right)=\left(\begin{array}{ccc}
0 & -x_{3} & x_{2} \\
x_{3} & 0 & -x_{1} \\
-x_{2} & x_{1} & 0
\end{array}\right) .
$$

We list, without proof, the basic results

$$
\begin{array}{ll}
\boldsymbol{\Lambda}(\mathbf{x}) \mathbf{y}=\mathbf{x} \times \mathbf{y}, & {[\boldsymbol{\Lambda}(\mathbf{x}), \mathbf{\Lambda}(\mathbf{y})]=\mathbf{\Lambda}(\mathbf{x} \times \mathbf{y}),} \\
\operatorname{Tr}(\boldsymbol{\Lambda}(\mathbf{x}) \boldsymbol{\Lambda}(\mathbf{y}))=-2 \mathbf{x} \cdot \mathbf{y}, & \boldsymbol{\Lambda}(\mathbf{x}) \boldsymbol{\Lambda}(\mathbf{y})=\mathbf{y} \mathbf{x}^{T}-\mathbf{x} \cdot \mathbf{y} \mathbf{1}_{3}, \\
(\mathbf{x} \times \mathbf{y}) \times \mathbf{z}=(\mathbf{x} \cdot \mathbf{z}) \mathbf{y}-(\mathbf{y} \cdot \mathbf{z}) \mathbf{x}, & \boldsymbol{\Lambda}(\mathbf{D} \mathbf{x})=\mathbf{D} \boldsymbol{\Lambda}(\mathbf{x}) \mathbf{D}^{T}, \\
\mathbf{x y}^{T}-\mathbf{y} \mathbf{x}^{T}=\mathbf{\Lambda}(\mathbf{y} \times \mathbf{x}), &
\end{array}
$$

where $\mathbf{1}_{3}$ is the unit $3 \times 3$ matrix. The last equation in (2.23) follows from the identity $\mathbf{D x} \times \mathbf{D y}=\mathbf{D}(\mathbf{x} \times \mathbf{y})$.

\subsection{Directors and reference configurations}

With respect to $s$, the matrix $\mathbf{D}(t, s)$ is line continuous and $\mathbf{D}_{\lambda}(t, v)$ is the limit of $\mathbf{D}$ at $v$ along $\lambda$. We set $\mathbf{D}(t, v)$ to be the matrix describing the rigid body frame at $v$. As mentioned in the introduction, there is a set of rotation matrices $\mathbf{X}(v, \lambda) \in \mathrm{SO}(3)$ for each line $\lambda$ such that (1.3) holds. This is equivalent to

$$
\mathbf{D}_{\lambda}(t, v)=\mathbf{D}(t, v) \mathbf{X}(v, \lambda)
$$

Differentiating the matrix $\mathbf{D}(t, s)$ with respect to $t$ and $s$ gives the angular velocity $\underline{w}$ and angular strain $\underline{u}_{D}$, respectively. These are given in 
the fixed basis by

$$
\Lambda\left(\mathbf{w}^{e}\right)=\dot{\mathbf{D}} \mathbf{D}^{T}, \quad \Lambda\left(\mathbf{u}_{D}^{e}\right)=\mathbf{D}^{\prime} \mathbf{D}^{T}
$$

respectively. Note that (2.23) implies that the angular velocity and angular strain in the director basis are given by $\Lambda\left(\mathbf{w}^{d}\right)=\mathbf{D}^{T} \dot{\mathbf{D}}$ and $\Lambda\left(\mathbf{u}_{D}^{d}\right)=$ $\mathbf{D}^{T} \mathbf{D}^{\prime}$ since

$$
\boldsymbol{\Lambda}\left(\mathbf{w}^{d}\right)=\boldsymbol{\Lambda}\left(\mathbf{D}^{T} \mathbf{w}^{e}\right)=\mathbf{D}^{T} \boldsymbol{\Lambda}\left(\mathbf{w}^{e}\right) \mathbf{D}=\mathbf{D}^{T} \dot{\mathbf{D}} \mathbf{D}^{T} \mathbf{D}=\mathbf{D}^{T} \dot{\mathbf{D}}
$$

Given a Cosserat element in the net corresponding to a line $\lambda$, the Cosserat equations for most physical constitutive relations and no external forces and torques admit a stress-free static solution, given by $\underline{r}_{\lambda}(t, s)=\underline{r}_{0 \lambda}(s)$ and $\mathbf{D}_{\lambda}(t, s)=\mathbf{D}_{0 \lambda}(s)$ modulo Euclidean translations and rotations. This is used to define $\underline{u}_{0 \lambda}$ for the line $\lambda$ via

$$
\Lambda\left(\mathbf{u}_{0 \lambda}^{d}\right)=\mathbf{D}_{0 \lambda}^{T} \mathbf{D}_{0 \lambda}^{\prime}
$$

Given $\underline{u}_{0 \lambda}$ for all the lines $\lambda$, we construct the line continuous vector $\underline{u}_{0}$. Clearly, $\mathbf{u}_{0}^{d}=\mathbf{u}_{0}^{d}(s)$ is independent of time. The relative angular strain usually appears in the constitutive relations, for example, as in the Kirchhoff constitutive relations given in Section 3.1. Note that in general there does not exist a stress-free static solution for the entire Cosserat net due to the existence of loops (see below). Also note that the constitutive relations for a general Cosserat element may admit a continuum of stressfree solutions, for example, the string, or, for nonlinear constitutive relations, several discreet stress-free solutions. In such case, one simply chooses a particular stress-free solution.

We define the relative angular strain to be

$$
\underline{u}_{\text {strain }}=\underline{u}_{D}-\underline{u}_{0}
$$

so that if a solution given by $\mathbf{D}(t, s)=\mathbf{D}_{0}(s)$ exists, then it is relative angular strain free.

It is convenient to introduce a line continuous matrix called the reference configuration,

$$
\mathrm{C}: \mathrm{Y} \longmapsto \mathrm{SO}(3) \text {, }
$$


and node matrices $\mathbf{C}(v)$ such that

$$
\mathbf{C}_{\lambda}(v)=\mathbf{C}(v) \mathbf{X}(v, \lambda)
$$

at each node $v$ and define a change of variables from $\mathbf{D}$ to $\mathbf{A}$ by

$$
\mathbf{A}(t, s)=\mathbf{D}(t, s) \mathbf{C}(s)^{T}
$$

It is easy to show that $\mathbf{A}$ is continuous on $\mathbf{Y}$ and $\mathbf{A}_{\curlywedge}(t, v)=\mathbf{A}(t, v)$. By writing $\mathbf{D}$ as $\mathbf{D}(t, s)=\mathbf{A}(t, v) \mathbf{C}(s)$ with $\mathbf{C}$ independent of time, the dynamics of the director fields is encoded into the matrix $\mathbf{A}$ which is continuous on Y. It also proves to be a useful tool for analysing perturbations about the static solution, as in Section 6.

In terms of $\mathbf{C}$ and $\mathbf{A}$, one has

$$
\underline{w}=\underline{w}_{A^{\prime}} \quad \underline{u}_{D}=\underline{u}_{A}+\underline{u}_{C^{\prime}}
$$

where (2.25) and $\boldsymbol{\Lambda}\left(\mathbf{w}_{A}^{e}\right)=\dot{\mathbf{A}} \mathbf{A}^{T}, \boldsymbol{\Lambda}\left(\mathbf{u}_{A}^{e}\right)=\mathbf{A}^{\prime} \mathbf{A}^{T}$, and $\boldsymbol{\Lambda}\left(\mathbf{u}_{C}^{e}\right)=\mathbf{A C}^{\prime} \mathbf{C}^{T} \mathbf{A}^{T}$. The proof of (2.32) is trivial. Note that $\mathbf{u}_{C}^{d}$ given by $\Lambda\left(\mathbf{u}_{C}^{d}\right)=\mathbf{C}^{T} \mathbf{C}^{\prime}$ is independent of time. The relative angular strain may be written as

$$
\underline{u}_{\text {strain }}=\underline{u}_{A}+\underline{u}_{C}-\underline{u}_{0} \text {. }
$$

Given $\mathbf{X}$, there is an infinite number of choices for $\mathbf{C}$. For example, one choice, which is always available, is to use the initial director fields in $\mathbf{Y}$, setting $\mathbf{C}(s)=\mathbf{D}(0, s)$ in which case $\mathbf{A}(0, s)=\mathbf{1}_{3}$.

Another particular useful choice for $\mathbf{C}$ which, if it exists, has a physical interpretation, is given by $\underline{u}_{C}=\underline{u}_{0}$. This is called the strain-free reference configuration since it implies $\underline{u}_{\text {strain }}=\underline{u}_{A}$. Clearly, this is given by the solutions of the $\mathrm{ODE} \mathbf{C}^{\prime}(s)=\mathbf{C}(s) \boldsymbol{\Lambda}\left(\mathbf{u}_{0}^{d}(s)\right)$ subject to the junction conditions (2.30). Such a solution, if it exists, is unique up to a fixed rotation.

It is not always possible to construct such a C. For example, consider three straight elements each with $\mathbf{u}_{0}^{d}=0$. Attach them at right angles to each other in a bent triangle. This requires bending the elements. The elements must bend since otherwise they will form a triangle with three right angles. Hence, such a configuration cannot be stress-free.

Although the existence of a strain-free reference configuration is not always guaranteed, we do have the following two observations. 
LEMMA 2.2. If there exists a relative angular strain-free configuration $\left(\underline{u}_{\text {strain }}=\right.$ $0)$, then there exists a strain-free reference configuration $\left(\underline{u}_{C}=\underline{u}_{0}\right)$.

Proof. Simply, note that if $\mathbf{D}_{0}$ is a relative angular strain-free configuration, then setting $\mathbf{C}=\mathbf{D}_{0}$ gives

$$
\Lambda\left(\mathbf{u}_{C}^{d}\right)=\mathbf{C}^{T} \mathbf{C}^{\prime}=\mathbf{D}_{0}^{T} \mathbf{D}_{0}^{\prime}=\Lambda\left(\mathbf{u}_{D}^{d}\right)=\Lambda\left(\mathbf{u}_{0}^{d}\right) .
$$

We note that this is not true in the reverse; the existence of a strain-free reference configuration does not imply a relative angular strain-free configuration. For example, consider a single straight element forced into a loop with the two ends joined at $180^{\circ}$. This is not strain free but has a strain-free reference configuration given by $\mathrm{C}=\mathbf{1}_{3}$.

LEMMA 2.3. If the net is simply connected (no loops) and the endpoints are not constrained, then there exists a strain-free reference configuration.

Proof. We can integrate the differential equation $\mathbf{C}^{\prime}=\mathbf{C} \boldsymbol{\Lambda}\left(\mathbf{u}_{0}^{d}\right)$ along each line and construct $\mathbf{C}$ globally using (2.30).

If there exists a stress-free static solution of Cosserat equations with the appropriate junction conditions given by $\underline{r}(t, s)=\underline{r}_{0}(s)$ and $\mathbf{D}(t, s)=$ $\mathbf{D}_{0}(s)$, then by Lemma 2.2 there exists a strain-free reference configuration $\mathbf{C}(s)=\mathbf{D}_{0}(s)$. The class of stress-free solutions given by Euclidean rotations and translations may be expressed by $\mathbf{A}(t, s)=\mathbf{A}_{0}$ and $\mathbf{r}^{e}(t, s)=$ $\mathbf{A}_{0} \mathbf{r}_{0}^{e}(s)+t \mathbf{k}_{1}+\mathbf{k}_{0}$, where $\mathbf{A}_{0}$ is a constant matrix and $\mathbf{k}_{0}$ and $\mathbf{k}_{1}$ are constant column vectors. This description will be used for the perturbations in Section 6.

\section{Cosserat equations for nets with point junctions}

In this section, we consider nets containing only point junctions. The configuration of such a net is described by the two maps

$$
\begin{array}{ll}
\underline{r}: \mathbb{R} \times \mathbf{Y} \longmapsto \mathcal{M}=\mathbb{R}^{3}, & \mathbf{A}: \mathbb{R} \times \mathbf{Y} \longmapsto \mathrm{SO}(3), \\
\underline{r}:(t, s) \longmapsto \underline{r}(t, s), & \mathbf{A}:(t, s) \longmapsto \mathbf{A}(t, s) .
\end{array}
$$

In the proceeding section, it is shown that $\mathbf{A}$ is continuous in $s$. This implies that the local angular velocity vector $\underline{w}(t, s)$ is continuous on Y. Since all junctions here are point junctions, then $\underline{r}$ is also continuous in $s$. Since all maps are assumed differentiable in time, the dependent variables $\underline{\dot{r}}, \underline{\ddot{r}}$, and $\underline{w}$ are all continuous. 
The nondimensional Cosserat equations for an interior point $s$ on a line $\lambda$ within the net at a time $t$ are

$$
\begin{gathered}
\mu \ddot{\ddot{r}}=\underline{n}^{\prime}+\underline{f}, \\
(\underline{\mathbf{I}}(\underline{w}))^{*}=\underline{m}^{\prime}+\kappa \underline{r}^{\prime} \times \underline{n}+\underline{l},
\end{gathered}
$$

where $\mu$ is mass-density parameter and $\underline{\mathbf{I}}$ is given in the director basis as

$$
\mathbf{I}^{d}=\left(\begin{array}{ccc}
I_{11} & I_{12} & 0 \\
I_{12} & I_{22} & 0 \\
0 & 0 & I_{33}
\end{array}\right)
$$

with $I_{33}=I_{22}+I_{11}$. In general, $\mu$ and the components of $\mathbf{I}^{d}$ are dependent on $s$. The contact force and torque acting on elements of the net are denoted by $\underline{n}$ and $\underline{m}$, respectively, and external forces and torques are $f$ and $\underline{l}$, respectively. The junction conditions are given by

$$
\begin{aligned}
M_{\nu} \ddot{\ddot{r}}(t, v) & =\underline{F}(t, v)-\sum_{\lambda} \Omega(v, \lambda) \underline{n}_{\lambda}(t, v), \\
\left(\underline{\mathbf{I}}_{v} \underline{w}(t, v)\right)^{\cdot} & =\underline{L}(t, v)-\sum_{\lambda} \Omega(v, \lambda) \underline{m}_{\lambda}(t, v) .
\end{aligned}
$$

THEOREM 3.1. If the contact forces and torques are hyperelastic and the external forces and torques are conservative, then (3.2), (3.3), (3.5), and (3.6) follow as stationary points of the action functional

$$
\begin{aligned}
\mathcal{S}= & \int_{\mathbb{R} \times \mathbf{Y}}\left(\frac{1}{2} \mu \underline{\dot{r}} \cdot \underline{\dot{r}}+\frac{1}{2} \kappa^{-1} \underline{w} \cdot \underline{\mathbf{I}}(\underline{w})-\mathcal{V}\left(\mathbf{v}^{d}, \mathbf{u}_{D}^{d}\right)-\varphi(\underline{r}, \mathbf{A})\right) d t d s \\
& +\sum_{v \in \mathcal{N}(\mathbf{Y})} \int_{\mathbb{R}}\left(\frac{1}{2} M_{v} \underline{\dot{r}} \cdot \underline{\dot{r}}+\frac{1}{2} \kappa^{-1} \underline{w} \cdot \underline{\mathbf{I}}_{v}(\underline{w})-\varphi_{v}(\underline{r}, \mathbf{A})\right) d t
\end{aligned}
$$

under constrained variations which preserve (2.25) and $\mathbf{v}^{d}=\mathbf{D}^{T} \mathbf{r}^{e^{\prime}}$, where the external forces and torques are derived from the potentials $\varphi$ and $\varphi_{\nu}$,

$$
\begin{array}{rlrl}
\mathbf{f}^{e} & =-\frac{\partial \varphi}{\partial \mathbf{r}^{e}}, & \mathbf{1}^{e}=2 \kappa \boldsymbol{\Lambda}^{-1}\left(\frac{\partial \varphi}{\partial \mathbf{A}} \mathbf{A}^{T}\right), \\
\mathbf{F}^{e}=-\frac{\partial \varphi_{v}}{\partial \mathbf{r}^{e}}, & \mathbf{L}^{e}=2 \kappa \boldsymbol{\Lambda}^{-1}\left(\frac{\partial \varphi_{v}}{\partial \mathbf{A}} \mathbf{A}^{T}\right),
\end{array}
$$


and the contact forces and torques are derived from the hyperelastic potential $\mho$

$$
\mathbf{n}^{d}=\frac{\partial \mho}{\partial \mathbf{v}^{d}}, \quad \mathbf{m}^{d}=\kappa^{-1} \frac{\partial \mho}{\partial \mathbf{u}_{D}^{d}} .
$$

When differentiating a scalar by a column vector or matrix, we use the notation

$$
\frac{\partial \varphi}{\partial \mathbf{x}}=\frac{\partial \varphi}{\partial \mathbf{x}_{i}} \mathbf{e}_{i}, \quad \frac{\partial \varphi}{\partial \mathbf{A}}=\frac{\partial \varphi}{\partial \mathbf{A}_{i j}} \mathbf{e}_{i} \mathbf{e}_{j}^{T} .
$$

Before proving this theorem, some preliminary variations with respect to $\mathbf{A}$ are derived.

LEMMA 3.2. If $\mathbf{A}$ is varied alone by the operator $\delta$ such that $\delta \mathbf{C}=0, \delta \mathbf{I}^{d}=0$, $\delta \mathbf{w}^{e}=0, \delta \boldsymbol{\alpha}=0$, and $\delta \boldsymbol{\beta}=0$, then

$$
\begin{aligned}
& \delta\left(\boldsymbol{\alpha} \cdot \boldsymbol{\Lambda}^{-1}\left(\dot{\mathbf{A}} \mathbf{A}^{T}\right)\right)=(\boldsymbol{\alpha} \cdot \delta \mathbf{a})^{\cdot}-\left(\dot{\boldsymbol{\alpha}}+\boldsymbol{\alpha} \times \boldsymbol{\Lambda}^{-1}\left(\dot{\mathbf{A}} \mathbf{A}^{T}\right)\right) \cdot \delta \mathbf{a}, \\
& \delta\left(\boldsymbol{\beta} \cdot \boldsymbol{\Lambda}^{-1}\left(\mathbf{D}^{T} \mathbf{D}^{\prime}\right)\right)=(\mathbf{D} \boldsymbol{\beta} \cdot \delta \mathbf{a})^{\prime}-(\mathbf{D} \boldsymbol{\beta})^{\prime} \cdot \delta \mathbf{a}, \\
& \delta\left(\mathbf{w}^{e} \cdot \mathbf{I}^{e} \mathbf{w}^{e}\right)=\mathbf{I}^{e} \mathbf{w}^{e} \times \mathbf{w}^{e} \cdot \delta \mathbf{a}, \\
& \delta \varphi(\mathbf{A})=2 \boldsymbol{\Lambda}^{-1}\left(\frac{\partial \varphi}{\partial \mathbf{A}} \mathbf{A}^{T}\right) \cdot \delta \mathbf{a},
\end{aligned}
$$

where $\mathbf{\Lambda}(\delta \mathbf{a})=\delta \mathbf{A} \mathbf{A}^{T}, \mathbf{D}=\mathbf{A C}$, and $\mathbf{I}^{e}=\mathbf{D I}^{d} \mathbf{D}^{T}$.

Proof. Since $\delta \mathbf{A} \mathbf{A}^{T}+\mathbf{A} \delta \mathbf{A}^{T}=0$ and $\mathbf{A}^{\prime} \mathbf{A}^{T}+\mathbf{A} \mathbf{A}^{T^{\prime}}=0$,

$$
\begin{aligned}
& \delta\left(\boldsymbol{\alpha} \cdot \boldsymbol{\Lambda}^{-1}\left(\dot{\mathbf{A}} \mathbf{A}^{T}\right)\right) \\
& =\boldsymbol{\alpha} \cdot \boldsymbol{\Lambda}^{-1}\left(\delta \dot{\mathrm{A}} \mathbf{A}^{T}\right)+\boldsymbol{\alpha} \cdot \boldsymbol{\Lambda}^{-1}\left(\dot{\mathrm{A}} \delta \mathbf{A}^{T}\right) \\
& =\left(\boldsymbol{\alpha} \cdot \boldsymbol{\Lambda}^{-1}\left(\delta \mathbf{A} \mathbf{A}^{T}\right)\right)^{\cdot}-\dot{\boldsymbol{\alpha}} \cdot \boldsymbol{\Lambda}^{-1}\left(\delta \mathbf{A} \mathbf{A}^{T}\right)-\boldsymbol{\alpha} \cdot \boldsymbol{\Lambda}^{-1}\left(\delta \mathbf{A} \dot{\mathbf{A}}^{T}\right)+\boldsymbol{\alpha} \cdot \boldsymbol{\Lambda}^{-1}\left(\dot{\mathbf{A}} \delta \mathbf{A}^{T}\right) \\
& =(\boldsymbol{\alpha} \cdot \delta \mathbf{a})^{\cdot}-\dot{\boldsymbol{\alpha}} \cdot \delta \mathbf{a}-\boldsymbol{\alpha} \cdot \boldsymbol{\Lambda}^{-1}\left(\boldsymbol{\Lambda}(\delta \mathbf{a}) \mathbf{A} \dot{\mathbf{A}}^{T}\right)-\boldsymbol{\alpha} \cdot \boldsymbol{\Lambda}^{-1}\left(\dot{\mathbf{A}} \mathbf{A}^{T} \boldsymbol{\Lambda}(\delta \mathbf{a})\right) \\
& =(\boldsymbol{\alpha} \cdot \delta \mathbf{a})^{\cdot}-\dot{\boldsymbol{\alpha}} \cdot \delta \mathbf{a}+\boldsymbol{\alpha} \cdot \boldsymbol{\Lambda}^{-1}\left(\boldsymbol{\Lambda}(\delta \mathbf{a}) \boldsymbol{\Lambda}\left(\boldsymbol{\Lambda}^{-1}\left(\dot{\mathbf{A}} \mathbf{A}^{T}\right)\right)-\boldsymbol{\Lambda}\left(\boldsymbol{\Lambda}^{-1}\left(\dot{\mathbf{A}} \mathbf{A}^{T}\right)\right) \boldsymbol{\Lambda}(\delta \mathbf{a})\right) \\
& =(\boldsymbol{\alpha} \cdot \delta \mathbf{a})^{\cdot}-\dot{\boldsymbol{\alpha}} \cdot \delta \mathbf{a}+\boldsymbol{\alpha} \cdot \delta \mathbf{a} \times \boldsymbol{\Lambda}^{-1}\left(\dot{\mathbf{A}} \mathbf{A}^{T}\right) .
\end{aligned}
$$


204 The dynamics of Cosserat nets

Since $\delta \mathbf{D}=\delta \mathbf{A C}, \boldsymbol{\Lambda}(\delta \mathbf{a})=\delta \mathbf{D} \mathbf{D}^{T}$. Furthermore, $\delta \mathbf{D D}^{T}+\mathbf{D} \delta \mathbf{D}^{T}=0$ and $\mathbf{D}^{\prime} \mathbf{D}^{T}+\mathbf{D} \mathbf{D}^{T^{\prime}}=0$, so

$$
\begin{aligned}
\delta\left(\boldsymbol{\beta} \cdot \boldsymbol{\Lambda}^{-1}\left(\mathbf{D}^{T} \mathbf{D}^{\prime}\right)\right) & \boldsymbol{\beta} \cdot \boldsymbol{\Lambda}^{-1}\left(\mathbf{D}^{T}(\delta \mathbf{D})^{\prime}\right)+\boldsymbol{\beta} \cdot \boldsymbol{\Lambda}^{-1}\left(\delta \mathbf{D}^{T} \mathbf{D}^{\prime}\right) \\
= & \left(\boldsymbol{\beta} \cdot \boldsymbol{\Lambda}^{-1}\left(\mathbf{D}^{T} \delta \mathbf{D}\right)\right)^{\prime}-\boldsymbol{\beta}^{\prime} \cdot \boldsymbol{\Lambda}^{-1}\left(\mathbf{D}^{T} \delta \mathbf{D}\right) \\
& -\boldsymbol{\beta} \cdot \boldsymbol{\Lambda}^{-1}\left(\mathbf{D}^{T^{\prime}} \delta \mathbf{D}\right)+\boldsymbol{\beta} \cdot \boldsymbol{\Lambda}^{-1}\left(\delta \mathbf{D}^{T} \mathbf{D}^{\prime}\right) \\
= & \left(\mathbf{D} \boldsymbol{\beta} \cdot \boldsymbol{\Lambda}^{-1}\left(\mathbf{D} \mathbf{D}^{T} \delta \mathbf{D} \mathbf{D}^{T}\right)\right)^{\prime}-\mathbf{D} \boldsymbol{\beta}^{\prime} \cdot \boldsymbol{\Lambda}^{-1}\left(\mathbf{D D}^{T} \delta \mathbf{D D}^{T}\right) \\
& -\mathbf{D} \boldsymbol{\beta} \cdot \boldsymbol{\Lambda}^{-1}\left(\mathbf{D} \mathbf{D}^{T^{\prime}} \delta \mathbf{D} \mathbf{D}^{T}\right)+\mathbf{D} \boldsymbol{\beta} \cdot \boldsymbol{\Lambda}^{-1}\left(\mathbf{D} \delta \mathbf{D}^{T} \mathbf{D}^{\prime} \mathbf{D}^{T}\right) \\
= & \left(\mathbf{D} \boldsymbol{\beta} \cdot \boldsymbol{\Lambda}^{-1} \boldsymbol{\Lambda}(\delta \mathbf{a})\right)^{\prime}-\mathbf{D} \boldsymbol{\beta}^{\prime} \cdot \boldsymbol{\Lambda}^{-1} \boldsymbol{\Lambda}(\delta \mathbf{a}) \\
& -\mathbf{D} \boldsymbol{\beta} \cdot \boldsymbol{\Lambda}^{-1}\left(\mathbf{D} \mathbf{D}^{T^{\prime}} \delta \mathbf{D} \mathbf{D}^{T}\right)+\mathbf{D} \boldsymbol{\beta} \cdot \boldsymbol{\Lambda}^{-1}\left(\mathbf{D} \delta \mathbf{D}^{T} \mathbf{D}^{\prime} \mathbf{D}^{T}\right) \\
= & (\mathbf{D} \boldsymbol{\beta} \cdot \delta \mathbf{a})^{\prime}-\mathbf{D} \boldsymbol{\beta}^{\prime} \cdot \delta \mathbf{a}+\mathbf{D} \boldsymbol{\beta} \cdot \boldsymbol{\Lambda}^{-1}\left(\mathbf{D}^{\prime} \mathbf{D}^{T} \delta \mathbf{D} \mathbf{D}^{T}\right) \\
& -\mathbf{D} \boldsymbol{\beta} \cdot \boldsymbol{\Lambda}^{-1}\left(\delta \mathbf{D} \mathbf{D}^{T} \mathbf{D}^{\prime} \mathbf{D}^{T}\right) \\
= & (\mathbf{D} \boldsymbol{\beta} \cdot \delta \mathbf{a})^{\prime}-\mathbf{D} \boldsymbol{\beta}^{\prime} \cdot \delta \mathbf{a}+\mathbf{D} \boldsymbol{\beta} \cdot \boldsymbol{\Lambda}^{-1}\left(\boldsymbol{\Lambda}\left(\boldsymbol{\Lambda}^{-1}\left(\mathbf{D}^{\prime} \mathbf{D}^{T}\right)\right) \boldsymbol{\Lambda}\left(\delta \mathbf{\Lambda}\left(\boldsymbol{\Lambda}^{-1}\left(\mathbf{D}^{\prime} \mathbf{D}^{T}\right)\right)\right)\right. \\
= & (\mathbf{D} \boldsymbol{\beta} \cdot \delta \mathbf{a})^{\prime}-\mathbf{D} \boldsymbol{\beta}^{\prime} \cdot \delta \mathbf{a}+\mathbf{D} \boldsymbol{\beta} \cdot \boldsymbol{\Lambda}^{-1}\left(\mathbf{D}^{\prime} \mathbf{D}^{T}\right) \times \delta \mathbf{a} \\
= & (\mathbf{D} \boldsymbol{\beta} \cdot \delta \mathbf{a})^{\prime}-\left(\mathbf{D} \boldsymbol{\beta}^{\prime}+\boldsymbol{\Lambda}^{-1}\left(\mathbf{D}^{\prime} \mathbf{D}^{T}\right) \times \mathbf{D} \boldsymbol{\beta}\right) \cdot \delta \mathbf{a} \\
= & (\mathbf{D} \boldsymbol{\beta} \cdot \delta \mathbf{a})^{\prime}-\left(\mathbf{D} \boldsymbol{\beta}^{\prime}+\mathbf{D}^{\prime} \boldsymbol{\beta}\right) \cdot \delta \mathbf{a} .
\end{aligned}
$$

Hence (3.12) is obtained. For (3.13),

$$
\begin{aligned}
\delta\left(\mathbf{w}^{e} \cdot \mathbf{I}^{e} \mathbf{w}^{e}\right) & =\frac{1}{2}\left(\mathbf{w}^{e} \cdot \delta \mathbf{D} \mathbf{I}^{d} \mathbf{D}^{T} \mathbf{w}^{e}+\mathbf{w}^{e} \cdot \mathbf{D} \mathbf{I}^{d} \delta \mathbf{D}^{T} \mathbf{w}^{e}\right) \\
& =\frac{1}{2}\left(\left(\mathbf{w}^{e} \times \delta \mathbf{a}\right) \cdot \mathbf{I}^{e} \mathbf{w}^{e}+\mathbf{w}^{e} \mathbf{a} \cdot \mathbf{I}^{e}\left(\mathbf{w}^{e} \times \delta \mathbf{a}\right)\right) \\
& =\mathbf{I}^{e} \mathbf{w}^{e} \times \mathbf{w}^{e} \cdot \delta \mathbf{a} .
\end{aligned}
$$

For (3.14), note first that $\Lambda(\mathbf{x})_{i j} \Lambda(\mathbf{y})_{i j}=2 \mathbf{x} \cdot \mathbf{y}$. Thus

$$
\begin{aligned}
\delta \varphi(\mathbf{A}) & =\frac{\partial \varphi}{\partial \mathbf{A}_{i j}} \delta \mathbf{A}_{i j}=\frac{\partial \varphi}{\partial \mathbf{A}_{i j}} \boldsymbol{\Lambda}(\delta \mathbf{a})_{i k} \mathbf{A}_{k j} \\
& =\boldsymbol{\Lambda}\left(\boldsymbol{\Lambda}^{-1}\left(\frac{\partial \varphi}{\partial \mathbf{A}} \mathbf{A}^{T}\right)\right)_{i k} \boldsymbol{\Lambda}(\delta \mathbf{a})_{i k}
\end{aligned}
$$

and the result follows. 
Proof of Theorem 3.1. To effect the required variations, we differentiate with respect to the components of vectors and use the fixed frame for $\mathbf{r}^{e}, \dot{\mathbf{r}}^{e}, \mathbf{r}^{e^{\prime}}, \mathbf{w}^{e}$ and the director frame for $\mathbf{u}_{D}^{d}, \mathbf{v}^{d}$. Using the Lagrange multipliers $\boldsymbol{\alpha}, \boldsymbol{\beta}, \boldsymbol{\gamma}$, and $\boldsymbol{\alpha}^{N}$ for the constraints, the action functional to be varied is given by

$$
\begin{aligned}
& \mathcal{S}=\mathcal{S}\left(\mathbf{r}^{e}, \dot{\mathbf{r}}^{e}, \mathbf{r}^{e^{\prime}}, \mathbf{A}, \dot{\mathbf{A}}, \mathbf{A}^{\prime}, \mathbf{w}^{e}, \mathbf{u}_{D^{d}}^{d}, \mathbf{v}^{d}, \boldsymbol{\alpha}, \boldsymbol{\beta}, \boldsymbol{\gamma}, \boldsymbol{\alpha}^{N}\right) \\
& =\int_{\mathbb{R} \times \mathbf{Y}}\left(\frac{1}{2} \mu \dot{\mathbf{r}}^{e} \cdot \dot{\mathbf{r}}^{e}+\frac{1}{2} \kappa^{-1} \mathbf{w}^{e} \cdot \mathbf{I}^{e}\left(\mathbf{w}^{e}\right)-\mho\left(\mathbf{v}^{d}, \mathbf{u}_{D}^{d}\right)-\varphi\left(\mathbf{r}^{e}, \mathbf{A}\right)\right. \\
& +\boldsymbol{\alpha} \cdot\left(\mathbf{w}^{e}-\boldsymbol{\Lambda}^{-1}\left(\dot{\mathbf{D}} \mathbf{D}^{T}\right)\right)+\boldsymbol{\beta} \cdot\left(\mathbf{u}_{D}^{d}-\boldsymbol{\Lambda}^{-1}\left(\mathbf{D}^{T} \mathbf{D}^{\prime}\right)\right) \\
& \left.+r \cdot\left(\mathbf{v}^{d}-\mathbf{D}^{T} \mathbf{r}^{e^{\prime}}\right)\right) d t d s \\
& +\sum_{v \in \mathcal{N}(\mathbf{Y})} \int_{\mathbb{R}}\left(\frac{1}{2} M \dot{\mathbf{r}}^{e} \cdot \dot{\mathbf{r}}^{e}+\frac{1}{2} \kappa^{-1} \mathbf{w}^{e} \cdot \mathbf{I}_{v}^{e}\left(\mathbf{w}^{e}\right)-\varphi_{v}\left(\mathbf{r}^{e}, \mathbf{A}\right)\right. \\
& \left.+\boldsymbol{\alpha}^{N} \cdot\left(\mathbf{w}^{e}-\boldsymbol{\Lambda}^{-1}\left(\dot{\mathbf{D}}^{T}\right)\right)\right) d t
\end{aligned}
$$

After applying the divergence theorem to the total variation, one has

$$
\begin{aligned}
& \delta \mathcal{S}=\int_{\mathbb{R} \times \mathbf{Y}}\left(\left(-\frac{\partial \varphi}{\partial \mathbf{r}^{e}}-\ddot{\mathbf{r}}^{e}+(\mathbf{D} \gamma)^{\prime}\right) \cdot \delta \mathbf{r}^{e}\right. \\
& +\left(2 \boldsymbol{\Lambda}^{-1}\left(\frac{\partial \varphi}{\partial \mathbf{A}} \mathbf{A}^{T}\right)+\kappa^{-1} \mathbf{I}^{e} \mathbf{w}^{e} \times \mathbf{w}^{e}\right. \\
& \left.+\dot{\boldsymbol{\alpha}}+\boldsymbol{\alpha} \times \boldsymbol{\Lambda}^{-1}\left(\dot{\mathrm{A}} \mathbf{A}^{T}\right)+(\mathbf{D} \boldsymbol{\beta})^{\prime}-\mathbf{D} \boldsymbol{\gamma} \times \mathbf{r}^{e^{\prime}}\right) \cdot \delta \mathbf{a} \\
& +\left(\kappa^{-1} \mathbf{I}^{e} \mathbf{w}^{e}+\boldsymbol{\alpha}\right) \cdot \delta \mathbf{w}^{e}+\left(-\frac{\partial \mho}{\partial \mathbf{u}_{D}^{d}}+\boldsymbol{\beta}\right) \cdot \delta \mathbf{u}_{D}^{d} \\
& +\left(-\frac{\partial \mho}{\partial \mathbf{v}^{d}}+\gamma\right) \cdot \delta \mathbf{v}^{d}+\delta \boldsymbol{\alpha} \cdot\left(\mathbf{w}^{e}-\boldsymbol{\Lambda}^{-1}\left(\dot{\mathbf{D}}^{T}\right)\right) \\
& \left.+\delta \boldsymbol{\beta} \cdot\left(\mathbf{u}_{D}^{d}-\boldsymbol{\Lambda}^{-1}\left(\mathbf{D}^{T} \mathbf{D}^{\prime}\right)\right)+\delta \boldsymbol{r} \cdot\left(\mathbf{v}^{d}-\mathbf{D}^{T} \mathbf{r}^{e^{\prime}}\right)\right) d t d s \\
& +\int_{\mathbb{R} \times \partial \mathbf{Y}}\left(-\mathbf{D} \boldsymbol{\gamma} \cdot \delta \mathbf{r}^{e}-\mathbf{D} \boldsymbol{\beta} \cdot \delta \mathbf{a}\right) d t \\
& +\sum_{\nu \in \mathcal{N}(\mathbf{Y})} \int_{\mathbb{R}}\left(\left(-\frac{\partial \varphi_{v}}{\partial \mathbf{r}^{e}}-M_{\nu} \ddot{\mathbf{r}}^{e}\right) \cdot \delta \mathbf{r}^{e}\right.
\end{aligned}
$$




$$
\begin{aligned}
& +\left(2 \boldsymbol{\Lambda}^{-1}\left(\frac{\partial \varphi_{v}}{\partial \mathbf{A}} \mathbf{A}^{T}\right)+\mathcal{\kappa}^{-1} \mathbf{I}_{\nu}^{e} \mathbf{w}^{e} \times \mathbf{w}^{e}\right. \\
& \left.+\dot{\boldsymbol{\alpha}}^{N}+\boldsymbol{\alpha}^{N} \times \mathbf{w}^{e}\right) \cdot \delta \mathbf{a}+\left(\mathcal{\kappa}^{-1} \mathbf{I}_{\nu}^{e} \mathbf{w}^{e}+\boldsymbol{\alpha}^{N}\right) \cdot \delta \mathbf{w}^{e} \\
& \left.+\delta \boldsymbol{\alpha}^{N} \cdot\left(\mathbf{w}^{e}-\boldsymbol{\Lambda}^{-1}\left(\dot{\mathbf{D}}^{T}\right)\right)\right) d t .
\end{aligned}
$$

As well as generating the constraints $\mathbf{w}^{e}=\boldsymbol{\Lambda}^{-1}\left(\dot{\mathbf{D}} \mathbf{D}^{T}\right), \mathbf{u}_{D}^{d}=\boldsymbol{\Lambda}^{-1}\left(\mathbf{D}^{T} \mathbf{D}^{\prime}\right)$, and $\mathbf{v}^{d}=\mathbf{D}^{T} \mathbf{r}^{e^{\prime}}$, the Lagrange multipliers evaluate to $\boldsymbol{\alpha}=-\mathcal{K}^{-1} \mathbf{I}^{e} \mathbf{W}^{e}, \boldsymbol{\alpha}^{N}=$ $-\mathcal{K}^{-1} \mathbf{I}_{\nu}^{e} \mathbf{w}^{e}, \boldsymbol{\beta}=\partial \boldsymbol{U} / \partial \mathbf{u}_{D}^{d}=\mathcal{K}^{-1} \mathbf{m}^{d}$, and $\boldsymbol{\gamma}=\partial \boldsymbol{U} / \partial \mathbf{v}^{d}=\mathbf{n}^{d}$. Substituting these into the coefficient of $\delta \mathbf{r}^{e}$ gives (3.2). Substituting these into the coefficient of $\delta$ a gives

$$
2 \boldsymbol{\Lambda}^{-1}\left(\frac{\partial \varphi}{\partial \mathbf{A}} \mathbf{A}^{T}\right)-\mathcal{\kappa}^{-1}\left(\mathbf{I}^{e} \mathbf{w}^{e}\right)^{\cdot}+\mathcal{\kappa}^{-1}\left(\mathbf{D m}^{d}\right)^{\prime}-\mathbf{D n}^{d} \times \mathbf{r}^{e^{\prime}}=0,
$$

hence (3.3). The junction conditions (3.5) and (3.6) follow by decomposing $\int_{\partial Y}$ as in Section 2.1.

\subsection{Kirchhoff constitutive relations}

For a net composed of rods, wires, or slender beams undergoing small displacements from a reference configuration, the Kirchhoff constitutive relations provide an adequate description of elastic properties in terms of a few elastic moduli. The contact forces and torques are then given as

$$
\underline{n}=\underline{\mathbf{K}}\left(\underline{v}-\underline{d}_{3}\right), \quad \underline{m}=\underline{\mathbf{J}}\left(\underline{u}_{D}-\underline{u}_{0}\right)
$$

with $\underline{v}=\underline{r}^{\prime}$,

$$
\begin{gathered}
\mathbf{J}^{d}=R_{J}\left(\begin{array}{ccc}
I_{11} & I_{12} & 0 \\
I_{12} & I_{22} & 0 \\
0 & 0 & x I_{33}
\end{array}\right), \quad \mathbf{K}^{d}=R_{K}(s)\left(\begin{array}{ccc}
x & 0 & 0 \\
0 & x & 0 \\
0 & 0 & 1
\end{array}\right), \\
R_{J}=\frac{E \rho_{0}}{E_{0} \rho}, \quad R_{K}(s)=\frac{E A(s)}{E_{0} A_{0}}, \quad x=\frac{G}{E} .
\end{gathered}
$$

Here, $E$ and $G$ are the Young's and shear moduli of elasticity, $\rho$ is the density, and $A(s)$ is the cross sectional area of the slender elastic components of the net. The quantities $E_{0}, A_{0}$, and $\rho_{0}$ are rescaling constants, defined in Section 5. 
These constitutive relations follow from the hyperelastic potential

$$
\mathcal{U}\left(\mathbf{v}^{d}, \mathbf{u}_{D}^{d}\right)=\frac{1}{2}\left(\mathbf{v}^{d}-\mathbf{e}_{3}\right)^{T} \mathbf{K}^{d}\left(\mathbf{v}^{d}-\mathbf{e}_{3}\right)+\frac{1}{2}\left(\mathbf{u}_{D}^{d}-\mathbf{u}_{0}^{d}\right)^{T} \mathbf{J}^{d}\left(\mathbf{u}_{D}^{d}-\mathbf{u}_{0}^{d}\right)
$$

\section{Cosserat nets with extended junctions}

In this section, the variational approach is generalised to Cosserat nets containing extended junctions. As before the configuration of such a Cosserat net is encoded into the maps

$$
\begin{array}{ll}
\underline{r}: \mathbb{R} \times \mathbf{Y} \longmapsto \mathbb{R}^{3}, & \mathbf{A}: \mathbb{R} \times \mathbf{Y} \longmapsto \mathrm{SO}(3), \\
\underline{r}:(t, s) \longmapsto \underline{r}(t, s), & \mathbf{A}:(t, s) \longmapsto \mathbf{A}(t, s),
\end{array}
$$

with the map $\mathbf{A}$ is continuous, so that $\underline{w}(t, s)$ is continuous. However, as mentioned in the introduction, with extended junctions, the map $\underline{r}$ is only line continuous with the discontinuities at the nodes given by (1.1)

$$
\underline{r}_{\lambda}(t, v)=\underline{r}(t, v)+\underline{\xi}(t, v, \lambda)
$$

where $\xi^{d}(\nu, \lambda)$ is independent of $t$. The dynamic equations for this Cosserat net are (3.2) and (3.3) together with the junction conditions

$$
\begin{aligned}
& M_{\nu} \underline{\ddot{r}}(t, v)=\underline{F}(t, v)-\sum_{\lambda} \Omega(v, \lambda) \underline{n}_{\lambda}(t, v), \\
&\left(\underline{\mathbf{I}}_{v} \underline{w}(t, v)\right)^{\cdot}=\underline{L}(t, v)-\sum_{\lambda} \Omega(v, \lambda)\left(\underline{m}_{\lambda}(t, v)+\kappa \underline{\xi}(t, v, \lambda) \times \underline{n}_{\lambda}(t, v)\right) .
\end{aligned}
$$

Here, $\underline{F}(t, v)$ is the total external force on the rigid body at node $v$ and $\underline{L}(t, v)$ the total external torque on node $v$ about the centre of mass $\underline{r}(t, v)$. For example, if one is given the external forces and torques at the line ends, say, $\underline{F}_{\lambda}(t, v)$ and $\underline{L}_{\lambda}(t, v)$, respectively, then

$$
\begin{aligned}
& \underline{F}(t, v)=\sum_{\lambda} \underline{F}_{\lambda}(t, v), \\
& \underline{L}(t, v)=\sum_{\lambda} \underline{L}_{\lambda}(t, v)+\underline{\xi}(t, v, \lambda) \times \underline{F}_{\lambda}(t, v) .
\end{aligned}
$$

Once the motion and rotation of the centroid $\underline{r}(t, v)$, and $\mathbf{A}(t, v)$ are calculated, one can use (4.2) to derive the motions of the endpoints $\underline{r}_{\lambda}(t, v)$. 
THEOREM 4.1. If the constitutive relations are hyperelastic and the external forces and torques are conservative, then (4.3) and (4.4) can be derived from the variations of the action

$$
\begin{aligned}
\mathcal{S}= & \int_{\mathbb{R} \times \mathbf{Y}}\left(\frac{1}{2} \underline{\dot{r}}_{\lambda} \cdot \underline{\underline{r}}_{\lambda}+\frac{1}{2} \kappa^{-1} \underline{w} \cdot \underline{\mathbf{I}}(\underline{w})-\mathcal{V}\left(\mathbf{v}_{\lambda}^{d}, \mathbf{u}_{D}^{d}\right)-\varphi\left(\underline{r}_{\lambda}, \mathbf{A}\right)\right) d t d s \\
& +\sum_{\nu \in \mathcal{N}(\mathbf{Y})} \int_{\mathbb{R}}\left(\frac{1}{2} M_{\nu} \underline{\dot{r}}_{v} \cdot \underline{\dot{r}}_{v}+\frac{1}{2} \kappa^{-1} \underline{w} \cdot \underline{\mathbf{I}}_{v}(\underline{w})-\varphi_{\nu}\left(\underline{r}_{v}, \mathbf{A}\right)\right) d t
\end{aligned}
$$

that maintain the constraints (2.25), $\mathbf{v}^{d}=\mathbf{D}^{T} \mathbf{r}^{e}$, and (4.2), where $\underline{r}_{v}(t)=\underline{r}(t, v)$. The external forces and torques and contact forces and torques are given by (3.8) and (3.9).

Proof. The proof of this theorem is an extension to the proof of Theorem 3.1. As well as the Lagrange multipliers $\boldsymbol{\alpha}, \boldsymbol{\beta}, \boldsymbol{\gamma}, \boldsymbol{\alpha}^{N}$, we introduce another $\boldsymbol{\theta}_{v, \lambda}$ for the constraint (4.2) and write $\boldsymbol{\xi}_{v, \lambda}^{d}=\boldsymbol{\xi}^{d}(v, \lambda)$. Thus the complete action becomes

$$
\begin{aligned}
\mathcal{S}= & \mathcal{S}\left(\mathbf{r}_{\lambda}^{e}, \dot{\mathbf{r}}_{\lambda}^{e}, \mathbf{r}_{\lambda}^{e \ell}, \mathbf{r}_{v}^{e}, \dot{\mathbf{r}}_{v}^{e}, \mathbf{r}_{v}^{e l}, \mathbf{A}, \dot{\mathbf{A}}, \mathbf{A}^{\prime}, \mathbf{w}^{e}, \mathbf{u}_{D}^{d}, \mathbf{v}^{d}, \boldsymbol{\alpha}, \boldsymbol{\beta}, \boldsymbol{\gamma}, \boldsymbol{\alpha}^{N}, \boldsymbol{\theta}_{v, \lambda}\right) \\
= & \int_{\mathbb{R} \times \mathbf{Y}}\left(\frac{1}{2} \dot{\mathbf{r}}_{\lambda}^{e} \cdot \dot{\mathbf{r}}_{\lambda}^{e}+\frac{1}{2} \kappa^{-1} \mathbf{w}^{e} \cdot \mathbf{I}^{e}\left(\mathbf{w}^{e}\right)-\mathcal{V}\left(\mathbf{v}^{d}, \mathbf{u}_{D}^{d}\right)-\varphi\left(\mathbf{r}_{\lambda}^{e}, \mathbf{A}\right)\right. \\
& +\boldsymbol{\alpha} \cdot\left(\mathbf{w}^{e}-\boldsymbol{\Lambda}^{-1}\left(\dot{\mathbf{D}} \mathbf{D}^{T}\right)\right)+\boldsymbol{\beta} \cdot\left(\mathbf{u}_{D}^{d}-\boldsymbol{\Lambda}^{-1}\left(\mathbf{D}^{T} \mathbf{D}^{\prime}\right)\right) \\
& \left.+\boldsymbol{r} \cdot\left(\mathbf{v}^{d}-\mathbf{D}^{T} \mathbf{r}^{e^{\prime}}\right)\right) d t d s \\
& +\sum_{v \in \mathcal{N}(\mathbf{Y})} \int_{\mathbb{R}}\left(\frac{1}{2} M \dot{\mathbf{r}}^{e} \cdot \dot{\mathbf{r}}^{e}+\frac{1}{2} \kappa^{-1} \mathbf{w}^{e} \cdot \mathbf{I}_{v}^{e}\left(\mathbf{w}^{e}\right)-\varphi_{v}\left(\mathbf{r}^{e}, \mathbf{A}\right)\right. \\
& \left.\quad+\boldsymbol{\alpha}^{N} \cdot\left(\mathbf{w}^{e}-\Lambda^{-1}\left(\dot{\mathbf{D}} \mathbf{D}^{T}\right)\right)\right) d t \\
& +\sum_{(v, \lambda) \in \partial \mathbf{Y}} \int_{\mathbb{R}} \boldsymbol{\theta}_{v, \lambda} \cdot\left(\mathbf{r}_{\lambda}^{e}-\mathbf{r}_{v}^{e}-\mathbf{A} \xi_{v, \lambda}^{d}\right) d t .
\end{aligned}
$$

Applying the divergence theorem to the total variation yields

$$
\begin{aligned}
\delta \mathcal{S}=\int_{\mathbb{R} \times \mathbf{Y}}( & \left(-\frac{\partial \varphi}{\partial \mathbf{r}_{\mathcal{\lambda}}^{e}}-\ddot{\mathbf{r}}_{\mathcal{\lambda}}^{e}+(\mathbf{D} \boldsymbol{\gamma})^{\prime}\right) \cdot \delta \mathbf{r}_{\mathcal{\lambda}}^{e} \\
+ & \left(2 \boldsymbol{\Lambda}^{-1}\left(\frac{\partial \varphi}{\partial \mathbf{A}} \mathbf{A}^{T}\right)+\mathcal{\kappa}^{-1} \mathbf{I}^{e} \mathbf{w}^{e} \times \mathbf{w}^{e}+\dot{\boldsymbol{\alpha}}\right. \\
& \left.+\boldsymbol{\alpha} \times \boldsymbol{\Lambda}^{-1}\left(\dot{\mathbf{A}} \mathbf{A}^{T}\right)+(\mathbf{D} \boldsymbol{\beta})^{\prime}-\mathbf{D} \boldsymbol{\gamma} \times \mathbf{r}_{\mathcal{\lambda}}{ }^{\prime}\right) \cdot \delta \mathbf{a}
\end{aligned}
$$




$$
\begin{aligned}
& +\left(\kappa^{-1} \mathbf{I}^{e} \mathbf{w}^{e}+\boldsymbol{\alpha}\right) \cdot \delta \mathbf{w}^{e}+\left(-\frac{\partial \mho}{\partial \mathbf{u}_{D}^{d}}+\boldsymbol{\beta}\right) \cdot \delta \mathbf{u}_{D}^{d} \\
& +\left(-\frac{\partial \mho}{\partial \mathbf{v}^{d}}+\boldsymbol{r}\right) \cdot \delta \mathbf{v}^{d}+\delta \boldsymbol{\alpha} \cdot\left(\mathbf{w}^{e}-\Lambda^{-1}\left(\dot{\mathbf{D}} \mathbf{D}^{T}\right)\right) \\
& \left.+\delta \boldsymbol{\beta} \cdot\left(\mathbf{u}_{D}^{d}-\boldsymbol{\Lambda}^{-1}\left(\mathbf{D}^{T} \mathbf{D}^{\prime}\right)\right)+\delta \boldsymbol{r} \cdot\left(\mathbf{v}^{d}-\mathbf{D}^{T} \mathbf{r}_{\mathcal{\ell}}^{e^{\prime}}\right)\right) d t d s \\
& +\int_{\mathbb{R} \times \partial \mathbf{Y}}\left(-\mathbf{D} \boldsymbol{\gamma} \cdot \delta \mathbf{r}_{\mathcal{\lambda}}^{e}-\mathbf{D} \boldsymbol{\beta} \cdot \delta \mathbf{a}\right) d t \\
& +\sum_{v \in \mathcal{N}(\mathbf{Y})} \int_{\mathbb{R}}\left(\left(-\frac{\partial \varphi_{v}}{\partial \mathbf{r}_{v}^{e}}-M_{v} \ddot{r}_{v}^{e}\right) \cdot \delta \mathbf{r}_{v}^{e}\right. \\
& +\left(2 \boldsymbol{\Lambda}^{-1}\left(\frac{\partial \varphi_{v}}{\partial \mathbf{A}} \mathbf{A}^{T}\right)+\mathcal{K}^{-1} \mathbf{I}_{v}^{e} \mathbf{w}^{e} \times \mathbf{w}^{e}+\dot{\boldsymbol{\alpha}}^{N}+\boldsymbol{\alpha}^{N} \times \mathbf{w}^{e}\right) \cdot \delta \mathbf{a} \\
& \left.+\left(\mathcal{\kappa}^{-1} \mathbf{I}_{\nu}^{e} \mathbf{w}^{e}+\boldsymbol{\alpha}^{N}\right) \cdot \delta \mathbf{w}^{e}+\delta \boldsymbol{\alpha}^{N} \cdot\left(\mathbf{w}^{e}-\Lambda^{-1}\left(\dot{\mathbf{D}} \mathbf{D}^{T}\right)\right)\right) d t \\
& +\sum_{(v, \lambda) \in \partial \mathbf{Y}} \int_{\mathbb{R}} \delta \boldsymbol{\theta}_{v, \lambda} \cdot\left(\mathbf{r}_{\lambda}^{e}-\mathbf{r}_{v}^{e}-\mathbf{A} \boldsymbol{\xi}_{v, \lambda}^{d}\right)+\boldsymbol{\theta}_{v, \lambda} \cdot\left(\delta \mathbf{r}_{\lambda}^{e}-\delta \mathbf{r}_{v}^{e}-\delta \mathbf{A} \boldsymbol{\xi}_{v, \lambda}^{d}\right) d t .
\end{aligned}
$$

From the $\delta \mathbf{r}_{\mathcal{\lambda}}^{e}$ variation at node $v$, one obtains the multiplier

$$
\boldsymbol{\theta}_{v, \lambda}=\Omega(v, \lambda) \mathbf{D} \gamma=\Omega(v, \lambda) \mathbf{D n}_{\lambda}^{d}=\Omega(v, \lambda) \mathbf{n}_{\lambda}^{e}(v) .
$$

The $\delta \mathbf{r}_{v}^{e}$ variation therefore gives

$$
-M_{v} \ddot{\mathbf{r}}_{v}^{e}-\frac{\partial \varphi_{v}}{\partial \mathbf{r}_{v}^{e}}-\sum_{\lambda} \boldsymbol{\theta}_{v, \lambda}=0
$$

hence (4.3) follows.

The $\delta$ a variation gives

$$
\begin{aligned}
0= & \sum_{\lambda} \Omega(v, \lambda)(-\mathbf{D} \boldsymbol{\beta})+2 \boldsymbol{\Lambda}^{-1}\left(\frac{\partial \varphi_{v}}{\partial \mathbf{A}} \mathbf{A}^{T}\right)+\mathcal{\kappa}^{-1} \mathbf{I}_{\nu}^{e} \mathbf{w}^{e} \times \mathbf{w}^{e}+\dot{\boldsymbol{\alpha}}^{N}+\boldsymbol{\alpha}^{N} \times \mathbf{w}^{e} \\
& -\sum_{\lambda} \boldsymbol{\xi}_{v, \lambda}^{d} \times \boldsymbol{\theta}_{v, \lambda} \\
= & \mathbf{L}^{e}-\left(\mathbf{I}^{e} \mathbf{w}^{e}\right)^{\cdot}-\sum_{\lambda} \Omega(v, \lambda)\left(\mathbf{m}^{e}+\kappa \boldsymbol{\xi}_{v, \lambda}^{d} \times \mathbf{n}^{e}\right),
\end{aligned}
$$


TABLE 5.1

\begin{tabular}{lcl}
\hline Quantity & Symbol & Dimensions \\
\hline Reference time & $T_{0}$ & (time) \\
Reference length & $L_{0}$ & (length) \\
Reference area & $A_{0}$ & (length) $^{2}$ \\
Reference density & $\rho_{0}$ & (mass) $(\text { length) })^{-3}$ \\
Reference torque & $\mathcal{G}_{0}$ & (mass) $(\text { length) })^{2}(\text { time })^{-2}$ \\
\hline
\end{tabular}

TABLE 5.2

\begin{tabular}{llc}
\hline Quantity & Symbol = Definition & Dimensions \\
\hline Reference Young's modulus & $E_{0}=L_{0}^{2} T_{0}^{-2} \rho_{0}$ & ${\text { (mass })(\text { length })^{-1}(\text { time })^{-2}}^{-}$ \\
Scaling factor & $\kappa=E_{0} A_{0} L_{0} / \mathcal{G}_{0}$ & 1 \\
Scale free mass per length & $\mu(s)=\rho A(s) / \rho_{0} A_{0}$ & 1 \\
Scaling factor & $R_{J}=E \rho_{0} / E_{0} \rho$ & 1 \\
Scaling factor & $R_{K}(s)=E A(s) / E_{0} A_{0}$ & 1 \\
\hline
\end{tabular}

where we have used

$$
\boldsymbol{\theta}_{v, \lambda} \cdot \delta \mathbf{A} \boldsymbol{\xi}_{v, \lambda}^{d}=\boldsymbol{\theta}_{v, \lambda} \cdot \boldsymbol{\Lambda}(\delta \mathbf{a}) \mathbf{A} \xi_{v, \lambda}^{d}=\boldsymbol{\theta}_{v, \lambda} \cdot \delta \mathbf{a} \times \boldsymbol{\xi}_{v, \lambda}^{d}=\boldsymbol{\xi}_{v, \lambda}^{d} \times \boldsymbol{\theta}_{v, \lambda} \cdot \delta \mathbf{a}
$$

and hence (4.4).

\section{Nondimensionalisation}

To recover the fully dimensioned form of the Cosserat net equations with Kirchhoff constitutive relations, one needs to effect a number of rescalings.

Reference scalars with dimensions are chosen as shown in Table 5.1. These are used to define the quantities shown in Table 5.2.

The following variables are rescaled according to

$$
\begin{aligned}
& \breve{t}=t T_{0}, \quad \underline{\check{v}}=\underline{v}, \quad \underline{\check{K}}=\underline{\mathbf{K}} E_{0} A_{0}, \\
& \check{s}=s L_{0}, \quad \underline{\check{w}}=\underline{w} / T_{0}, \quad \check{M}_{v}=M_{v} \rho_{0} A_{0} L_{0} \text {, } \\
& \underline{\check{r}}=\underline{r} L_{0}, \quad \underline{\check{f}}=\underline{f} E_{0} A_{0} / L_{0}, \quad \underline{\check{I}}_{\nu}=\underline{\mathbf{I}}_{v} T_{0}^{2} \mathcal{G}_{0}, \\
& \underline{\check{n}}=\underline{n} E_{0} A_{0}, \quad \underline{\check{l}}=\underline{l} \mathcal{G}_{0} / L_{0}, \quad \underline{\breve{F}}=\underline{F} E_{0} A_{0}, \\
& \underline{\check{m}}=\underline{m} \mathcal{G}_{0}, \quad \underline{\check{\mathbf{I}}}=\underline{\mathbf{I}} T_{0}^{2} \mathcal{G}_{0} / L_{0}, \quad \underline{\check{L}}=\underline{L} \mathcal{G}_{0}, \\
& \underline{\check{u}}_{D}=\underline{u}_{D} / L_{0}, \quad \underline{\mathbf{J}}=\underline{\mathbf{J}} \mathcal{G}_{0} L_{0}, \quad \underline{\underline{\xi}}=\underline{\xi} L_{0} \text {. }
\end{aligned}
$$


Thus for any line $\lambda$ labelling an element of the net with stress-free length $L_{\text {Phys }}$, the material coordinate $s$ runs from $s_{\lambda}^{\min }$ to $s_{\lambda}^{\max }$ in the chosen orientation where $s_{\lambda}^{\max }-s_{\lambda}^{\min }=L_{\text {Phys }} / L_{0}$. Note that $\mathcal{\kappa}$ is no more than a convenient rescaling parameter and does not necessarily give any information about the relative flexibility of any element. Such information is encoded in the other parameters.

In terms of variables with physical dimensions, the general Cosserat net equations become

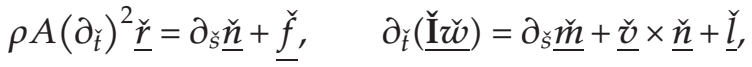

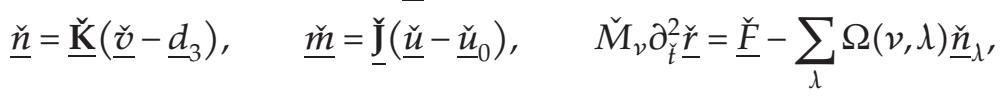

$$
\begin{aligned}
& \partial_{\breve{t}}\left(\check{I}_{v} \underline{\check{w}}\right)=\underline{L}_{-}-\sum_{\lambda} \Omega(v, \lambda)\left(\underline{\check{m}}_{\lambda}(t, v)+\underline{\breve{\xi}}^{(}(t, v, \lambda) \times \underline{\check{n}}_{\lambda}(t, v)\right)
\end{aligned}
$$

with

$$
\begin{aligned}
\underline{\mathbf{I}} & =\left(\begin{array}{ccc}
\check{I}_{11} & \check{I}_{12} & 0 \\
\check{I}_{12} & \check{I}_{22} & 0 \\
0 & 0 & \check{I}_{33}
\end{array}\right), \\
\underline{\mathbf{J}} & =\left(\begin{array}{ccc}
E \check{I}_{11} / \rho & E \check{I}_{12} / \rho & 0 \\
E \check{I}_{12} / \rho & E \check{I}_{22} / \rho & 0 \\
0 & 0 & G \check{I}_{33} / \rho
\end{array}\right), \\
\underline{\mathbf{\mathbf { K }}} & =\left(\begin{array}{ccc}
G A & 0 & 0 \\
0 & G A & 0 \\
0 & 0 & E A
\end{array}\right) .
\end{aligned}
$$

\section{Linearised Cosserat nets}

In this section, equations for linearised perturbations of a Cosserat net about a static strain-free solution are derived and the resulting system is reduced to an algebraic eigenproblem for its normal modes. We assume that the Cosserat net has Kirchhoff constitutive relations and a static stress-free solution given by $\underline{r}(t, s)=\underline{r}_{0}(s)$ and $\mathbf{D}(t, s)=\mathbf{D}_{0}(s)$. Let $\mathbf{C}(s)=\mathbf{D}_{0}(s)$ be the strain-free reference configuration. Let $\epsilon$ be a small perturbation parameter, and set

$$
\underline{r}(t, s)=\underline{r}_{0}(s)+\epsilon \underline{\hat{r}}(t, s), \quad \mathbf{A}(t, s)=\mathbf{1}_{3}+\epsilon \Lambda\left(\hat{\mathbf{a}}^{e}(t, s)\right),
$$

where $\hat{\mathbf{a}}^{e}$ are the components of the vector $\underline{\hat{a}}$ in the fixed basis. This vector represents the direction of the rotation of the unperturbed frame to give a perturbed frame. 
212 The dynamics of Cosserat nets

The components of $\underline{\hat{r}}$ and $\underline{\hat{a}}$ with respect to the $\underline{e}_{i}$ and $\underline{d}_{i}$ frames are given, as usual by (2.17), so that

$$
\hat{\mathbf{r}}^{e}=\mathbf{C} \hat{\mathbf{r}}^{d}+O(\epsilon), \quad \hat{\mathbf{a}}^{e}=\mathbf{C} \hat{\mathbf{a}}^{d}+O(\epsilon) .
$$

Note that $\hat{\mathbf{r}}^{d}$ represents the perturbed $\underline{r}$ in the $\underline{d}_{i}$ frame, not the perturbation of $\mathbf{r}^{d}$ which is given by

$$
\mathbf{r}^{d}=\mathbf{C}^{T} \mathbf{r}_{0}^{e}+\epsilon\left(\mathbf{C}^{T} \hat{\mathbf{r}}^{e}-\mathbf{C}^{T} \hat{\mathbf{a}}^{e} \times \mathbf{C}^{T} \mathbf{r}_{0}^{e}\right)
$$

THEOREM 6.1. The equations for the above perturbation are given by

$$
\begin{gathered}
\mu \underline{\hat{\hat{r}}}=\underline{u}_{D} \times \underline{\mathbf{K}}\left(\underline{\hat{r}}^{\prime}-\underline{\hat{a}} \times \underline{c}_{3}\right)+\underline{\mathbf{K}}\left(\underline{\hat{r}}^{\prime \prime}-\underline{u}_{D} \times \underline{\hat{r}}^{\prime}-\underline{\hat{a}}^{\prime} \times \underline{c}_{3}+\left(\underline{u}_{D} \times \underline{\hat{a}}\right) \times \underline{c}_{3}\right), \\
\underline{\mathbf{I}}(\underline{\ddot{\hat{\theta}}})=\underline{u}_{D} \times \underline{\mathbf{J}}(\underline{\hat{a}})+\underline{\mathbf{J}}\left(\underline{\hat{a}}^{\prime \prime}-\underline{u}_{D} \times \underline{\hat{a}}\right)+\kappa \underline{c}_{3} \times \underline{\mathbf{K}}\left(\underline{\hat{r}}^{\prime}-\underline{\hat{a}} \times \underline{c}_{3}\right),
\end{gathered}
$$

where $\underline{c}_{3}=\underline{r}_{0}$ and $\mathbf{r}_{0}^{e}=\mathbf{C e}_{3}$.

Proof. Since $\mathbf{n}^{e}=\mathbf{A C K} \mathbf{K}^{d}\left(\mathbf{C}^{T} \mathbf{A}^{T}\left(\mathbf{r}^{e^{\prime}}\right)-\mathbf{e}_{3}\right)$,

$$
\begin{aligned}
\mathbf{n}^{e} & =\mathbf{n}_{0}^{e}+\epsilon \hat{\mathbf{n}}^{e} \\
& =\left(1+\epsilon \boldsymbol{\Lambda}\left(\hat{\mathbf{a}}^{e}\right)\right) \mathbf{C} \mathbf{K}^{d}\left(\mathbf{C}^{T}\left(1-\epsilon \boldsymbol{\Lambda}\left(\hat{\mathbf{a}}^{e}\right)\right)\left(\mathbf{r}_{0}^{e \prime}+\epsilon \hat{\mathbf{r}}^{e}\right)-\mathbf{e}_{3}\right) .
\end{aligned}
$$

Since $\underline{r}_{0}(s)$ and $\mathbf{D}_{0}(s)=\mathbf{C}(s)$ yield a static stress-free solution, then $\mathbf{n}_{0}^{e}=0$ and so $\mathbf{r}_{0}^{e}=\mathrm{Ce}_{3}$ and

$$
\hat{\mathbf{n}}^{e}=\mathbf{C K}^{d} \mathbf{C}^{T}\left(\hat{\mathbf{r}}^{e^{\prime}}-\Lambda\left(\hat{\mathbf{a}}^{e}\right) \mathbf{r}_{0}^{e \prime}\right)=\mathbf{C K}^{d} \mathbf{C}^{T}\left(\hat{\mathbf{r}}^{{ }^{\prime}}-\hat{\mathbf{a}}^{e} \times \mathbf{c}_{3}^{e}\right) .
$$

Differentiating gives

$$
\begin{aligned}
\hat{\mathbf{n}}^{e \prime}= & \boldsymbol{\Lambda}\left(\mathbf{u}_{C}^{c}\right) \mathbf{C} \mathbf{K}^{d} \mathbf{C}^{T}\left(\hat{\mathbf{r}}^{e^{\prime}}-\hat{\mathbf{a}}^{e} \times \mathbf{c}_{3}^{e}\right)-\mathbf{C} \mathbf{K}^{d} \mathbf{C}^{T} \boldsymbol{\Lambda}\left(\mathbf{u}_{C}^{c}\right)\left(\hat{\mathbf{r}}^{e \prime}-\hat{\mathbf{a}}^{e} \times \mathbf{c}_{3}^{e}\right) \\
& +\mathbf{C} \mathbf{K}^{d} \mathbf{C}^{T}\left(\hat{\mathbf{r}}^{e \prime}-\hat{\mathbf{a}}^{e \prime} \times \mathbf{c}_{3}^{e}-\hat{\mathbf{a}}^{e} \times \boldsymbol{\Lambda}\left(\mathbf{u}_{C}^{c}\right) \mathbf{c}_{3}^{e}\right) \\
= & \mathbf{u}_{C}^{c} \times \mathbf{C} \mathbf{K}^{d} \mathbf{C}^{T}\left(\hat{\mathbf{r}}^{\prime \prime}-\hat{\mathbf{a}}^{e} \times \mathbf{c}_{3}^{e}\right) \\
& +\mathbf{C} \mathbf{K}^{d} \mathbf{C}^{T}\left(-\mathbf{u}_{C}^{c} \times \hat{\mathbf{r}}^{e \prime}+\hat{\mathbf{r}}^{e \prime}-\hat{\mathbf{a}}^{e \prime} \times \mathbf{c}_{3}^{e}+\left(\mathbf{u}_{C}^{c} \times \hat{\mathbf{a}}^{e}\right) \times \mathbf{c}_{3}^{e}\right),
\end{aligned}
$$

where $\boldsymbol{\Lambda}\left(\mathbf{u}_{C}^{c}\right)=\mathbf{C}^{\prime} \mathbf{C}^{T}$. Now, $\mathbf{A}=\mathbf{1}_{3}+O(\epsilon)$ hence $\mathbf{C K}^{d} \mathbf{C}^{T}=\mathbf{K}^{e}+O(\epsilon)$. One also has

$$
\boldsymbol{\Lambda}\left(\mathbf{u}_{D}^{e}\right)=\mathbf{D}^{\prime} \mathbf{D}^{T}=\mathbf{A}^{\prime} \mathbf{A}^{T}+\mathbf{A C}^{\prime} \mathbf{C}^{T} \mathbf{A}^{T}=\boldsymbol{\Lambda}\left(\mathbf{u}_{C}^{c}\right)+O(\epsilon),
$$


So

$$
\begin{aligned}
\hat{\mathbf{n}}^{e \prime}= & \mathbf{u}_{D}^{e} \times \mathbf{K}^{e}\left(\hat{\mathbf{r}}^{e^{\prime}}-\hat{\mathbf{a}}^{e} \times \mathbf{c}_{3}^{e}\right) \\
& +\mathbf{K}^{e}\left(\hat{\mathbf{r}}^{e^{\prime \prime}}-\mathbf{u}_{D}^{e} \times \hat{\mathbf{r}}^{e^{\prime}}-\hat{\mathbf{a}}^{e^{\prime}} \times \mathbf{c}_{3}^{e}+\left(\mathbf{u}_{D}^{e} \times \hat{\mathbf{a}}^{e}\right) \times \mathbf{c}_{3}^{e}\right) .
\end{aligned}
$$

Hence (6.4) follows. For (6.5), note that $\Lambda\left(\mathbf{w}^{e}\right)=\dot{\mathbf{A}} \mathbf{A}^{T}=\epsilon \Lambda\left(\dot{\hat{\mathbf{a}}}^{e}\right)+O\left(\epsilon^{2}\right)$. Hence $\mathbf{w}^{e}=\epsilon \dot{\hat{\mathbf{a}}}^{e}+O\left(\epsilon^{2}\right)$. Also $\mathbf{I}^{e}=\mathbf{C I}^{d} \mathbf{C}^{T}+O(\epsilon)$, hence $\left(\mathbf{I}^{e}\right)^{\cdot}=0+O(\epsilon)$. The left-hand side of (3.3) is given by $\left(\mathbf{I}^{e} \mathbf{w}^{e}\right)^{\cdot}=\epsilon \mathbf{I}^{e}\left(\ddot{\hat{\mathbf{a}}}^{e}\right)$. For the righthand side, we have

$$
\mathbf{r}^{\mathbf{r}^{\prime}} \times \mathbf{n}^{e}=\epsilon \mathbf{r}_{0}^{e} \times \hat{\mathbf{n}}^{e}+O\left(\epsilon^{2}\right)=\epsilon \mathbf{c}_{3}^{e} \times \mathbf{K}^{e}\left(\hat{\mathbf{r}}^{e \prime}-\hat{\mathbf{a}}^{e} \times \mathbf{c}_{3}^{e}\right)+O\left(\epsilon^{2}\right) .
$$

Since we are perturbing about a static strain-free solution $\mathbf{C}(s)=\mathbf{D}_{0}(s)$, then $\boldsymbol{\Lambda}\left(\mathbf{u}_{0}^{d}\right)=\mathbf{D}_{0}^{T} \mathbf{D}_{0}^{\prime}=\mathbf{C}^{T} \mathbf{C}^{\prime}=\boldsymbol{\Lambda}\left(\mathbf{u}_{C}^{d}\right)$, so $\underline{u}_{C}=\underline{u}_{0}$. From (2.32), we have $\underline{u}_{D}=\underline{u}_{A}+\underline{u}_{0}$ and from (3.22), $\underline{m}=\mathbf{J}\left(\underline{u}_{D}-\underline{u}_{0}\right)=\underline{u}_{A}$. Now, $\Lambda\left(\mathbf{u}_{A}^{e}\right)=\mathbf{A}^{\prime} \mathbf{A}^{T}=$ $\epsilon \Lambda\left(\hat{\mathbf{a}}^{e^{\prime}}\right)\left(1-\Lambda\left(\hat{\mathbf{a}}^{e}\right)\right)$ gives $\mathbf{u}_{A}^{e}=\epsilon \hat{\mathbf{a}}^{e^{-}}+O\left(\epsilon^{2}\right)$. Thus $\mathbf{m}^{e}=\epsilon \mathbf{J}^{e}\left(\hat{\mathbf{a}}^{e^{\prime}}\right)+O\left(\epsilon^{2}\right)$. Differentiating yields

$$
\mathbf{m}^{e^{\prime}}=\epsilon \mathbf{u}_{C}^{c} \times \mathbf{J}^{e}\left(\hat{\mathbf{a}}^{e \prime}\right)-\epsilon \mathbf{J}^{e}\left(\mathbf{u}_{C}^{c} \times \hat{\mathbf{a}}^{e^{\prime}}\right)+\epsilon \mathbf{J}^{e}\left(\hat{\mathbf{a}}^{e^{\prime \prime}}\right)+O\left(\epsilon^{2}\right)
$$

and putting this all together gives (6.5).

For simplicity, assume that the unstrained state of each Cosserat element is straight, so that $\mathbf{C}$ is constant along each Cosserat element, and $\underline{u}_{C}=0$. Thus

$$
\begin{aligned}
\mu \underline{\hat{r}} & =\underline{\mathbf{K}}\left(\underline{\hat{r}}^{\prime \prime}-\underline{\hat{a}}^{\prime} \times \underline{c}_{3}\right), \\
\underline{\mathbf{I}}(\underline{\hat{\hat{a}}}) & =\underline{\mathbf{J}}\left(\underline{\hat{a}}^{\prime \prime}\right)+\kappa \underline{c}_{3} \times \underline{\mathbf{K}}\left(\underline{\hat{r}}^{\prime}-\underline{\hat{a}} \times \underline{c}_{3}\right) .
\end{aligned}
$$

To analyse these equations, suppose there exist mode functions $\underline{\tilde{r}}(s)$ and $\underline{\tilde{a}}(s)$ such that

$$
\underline{\hat{r}}(t, s)=\sin (\omega t) \underline{\tilde{r}}(s), \quad \underline{\hat{a}}(t, s)=\sin (\omega t) \underline{\tilde{a}}(s) .
$$

Then

$$
\begin{aligned}
-\omega^{2} \mu \underline{\tilde{r}} & =\underline{\mathbf{K}}\left(\underline{\tilde{r}}^{\prime \prime}-\underline{\tilde{a}}^{\prime} \times \underline{c}_{3}\right), \\
-\omega^{2} \underline{\mathbf{I}}(\underline{\tilde{a}}) & =\underline{\mathbf{J}}\left(\underline{\tilde{a}}^{\prime \prime}\right)+\kappa \underline{c}_{3} \times \underline{\mathbf{K}}\left(\underline{r}^{\prime}-\underline{\hat{a}} \times \underline{c}_{3}\right) .
\end{aligned}
$$


214 The dynamics of Cosserat nets

To solve these equations, we express them in the director frame. Using (6.2) gives

$$
\begin{aligned}
-\omega^{2} \mu \tilde{\mathbf{r}}^{d} & =\mathbf{K}^{d}\left(\tilde{\mathbf{r}}^{d \prime \prime}-\tilde{\mathbf{a}}^{d \prime} \times \mathbf{e}_{3}\right), \\
-\omega^{2} \mathbf{I}^{d}\left(\tilde{\mathbf{a}}^{d}\right) & =\mathbf{J}^{d}\left(\tilde{\mathbf{a}}^{d \prime \prime}\right)+\kappa \mathbf{e}_{3} \times \mathbf{K}^{d}\left(\mathbf{r}^{d^{\prime}}-\tilde{\mathbf{a}}^{d} \times \mathbf{e}_{3}\right),
\end{aligned}
$$

since $\mathbf{C}^{T} \mathbf{c}_{3}^{e}=\mathbf{e}_{3}+O(\epsilon)$. With $\mathbf{I}^{d}$ diagonal, $\mathbf{J}^{d}$ and $\mathbf{K}^{d}$ are also diagonal and considerable simplification ensues

$$
\begin{aligned}
& 0=\omega^{2} \mu \tilde{\mathbf{r}}_{1}^{d}+K_{11}\left(\tilde{\mathbf{r}}_{1}^{d \prime \prime}-\tilde{\mathbf{a}}_{2}^{d \prime}\right), \\
& 0=\omega^{2} \mu \tilde{\mathbf{r}}_{2}^{d}+K_{22}\left(\tilde{\mathbf{r}}_{2}^{d \prime \prime}+\tilde{\mathbf{a}}_{1}^{d \prime}\right), \\
& 0=\omega^{2} \mu \tilde{\mathbf{r}}_{3}^{d}+K_{33} \tilde{\mathbf{r}}_{3}^{d \prime \prime}, \\
& 0=\omega^{2} I_{11} \tilde{\mathbf{a}}_{1}^{d}+J_{11} \tilde{\mathbf{a}}_{1}^{d \prime \prime}-\kappa K_{22}\left(\tilde{\mathbf{r}}_{2}^{d \prime}+\tilde{\mathbf{a}}_{1}^{d}\right), \\
& 0=\omega^{2} I_{22} \tilde{\mathbf{a}}_{2}^{d}+J_{22} \tilde{\mathbf{a}}_{2}^{d \prime \prime}+\kappa K_{11}\left(\tilde{\mathbf{r}}_{1}^{d \prime}-\tilde{\mathbf{a}}_{2}^{d}\right), \\
& 0=\omega^{2} I_{33} \tilde{\mathbf{a}}_{3}^{d}+J_{33} \tilde{\mathbf{a}}_{3}^{d \prime \prime} .
\end{aligned}
$$

For $\omega^{2} I_{11}>\kappa K_{22}$ and $\omega^{2} I_{22}>\kappa K_{11}$, these gives

$$
\begin{aligned}
\tilde{\mathbf{r}}_{1}^{d}= & B_{1}^{\lambda} \cos \left(\alpha_{1} s\right)+B_{2}^{\lambda} \sin \left(\alpha_{1} s\right)+B_{3}^{\lambda} \cos \left(\alpha_{2} s\right)+B_{4}^{\lambda} \sin \left(\alpha_{2} s\right), \\
\tilde{\mathbf{r}}_{2}^{d}= & B_{5}^{\lambda} \cos \left(\alpha_{3} s\right)+B_{6}^{\lambda} \sin \left(\alpha_{3} s\right)+B_{7}^{\lambda} \cos \left(\alpha_{4} s\right)+B_{8}^{\lambda} \sin \left(\alpha_{4} s\right), \\
\tilde{\mathbf{r}}_{3}^{d}= & B_{9}^{\lambda} \cos \left(\alpha_{5} s_{\curlywedge}\right)+B_{10}^{\lambda} \sin \left(\alpha_{5} s_{\curlywedge}\right), \\
\tilde{\mathbf{a}}_{1}^{d}= & \frac{\omega^{2} \mu-K_{22} \alpha_{3}^{2}}{K_{22} \alpha_{3}}\left(B_{5}^{\lambda} \sin \left(\alpha_{3} s\right)-B_{6}^{\lambda} \cos \left(\alpha_{3} s\right)\right) \\
& +\frac{\omega^{2} \mu-K_{22} \alpha_{4}^{2}}{K_{22} \alpha_{4}}\left(B_{7}^{\lambda} \sin \left(\alpha_{4} s\right)-B_{8}^{\lambda} \cos \left(\alpha_{4} s\right)\right), \\
\tilde{\mathbf{a}}_{2}^{d}= & \frac{\omega^{2} \mu-K_{11} \alpha_{1}^{2}}{K_{11} \alpha_{1}}\left(B_{1}^{\lambda} \sin \left(\alpha_{1} s\right)-B_{2}^{\lambda} \cos \left(\alpha_{1} s\right)\right) \\
& +\frac{\omega^{2} \mu-K_{11} \alpha_{2}^{2}}{K_{11} \alpha_{2}}\left(B_{3}^{\lambda} \sin \left(\alpha_{2} s\right)-B_{4}^{\lambda} \cos \left(\alpha_{2} s\right)\right), \\
\tilde{\mathbf{a}}_{3}^{d}= & B_{11}^{\lambda} \cos \left(\alpha_{6} s_{\curlywedge}\right)+B_{12}^{\lambda} \sin \left(\alpha_{6} s_{\curlywedge}\right),
\end{aligned}
$$


where

$$
\begin{aligned}
& \alpha_{1}^{2}=\frac{\omega^{2}}{2}\left(\frac{I_{22}}{J_{22}}+\frac{\mu}{K_{11}}\right)+\left(\frac{\omega^{4}}{4}\left(\frac{I_{22}}{J_{22}}-\frac{\mu}{K_{11}}\right)^{2}+\omega^{2} \frac{\kappa \mu}{J_{22}}\right)^{1 / 2}, \\
& \alpha_{2}^{2}=\frac{\omega^{2}}{2}\left(\frac{I_{22}}{J_{22}}+\frac{\mu}{K_{11}}\right)-\left(\frac{\omega^{4}}{4}\left(\frac{I_{22}}{J_{22}}-\frac{\mu}{K_{11}}\right)^{2}+\omega^{2} \frac{\kappa \mu}{J_{22}}\right)^{1 / 2}, \\
& \alpha_{3}^{2}=\frac{\omega^{2}}{2}\left(\frac{I_{11}}{J_{11}}+\frac{\mu}{K_{22}}\right)+\left(\frac{\omega^{4}}{4}\left(\frac{I_{11}}{J_{11}}-\frac{\mu}{K_{22}}\right)^{2}+\omega^{2} \frac{\kappa \mu}{J_{11}}\right)^{1 / 2}, \\
& \alpha_{4}^{2}=\frac{\omega^{2}}{2}\left(\frac{I_{11}}{J_{11}}+\frac{\mu}{K_{22}}\right)-\left(\frac{\omega^{4}}{4}\left(\frac{I_{11}}{J_{11}}-\frac{\mu}{K_{22}}\right)^{2}+\omega^{2} \frac{\kappa \mu}{J_{11}}\right)^{1 / 2}, \\
& \alpha_{5}^{2}=\frac{\omega^{2} \mu}{K_{33}}, \\
& \alpha_{6}^{2}=\frac{\omega^{2} I_{33}}{J_{33}} .
\end{aligned}
$$

If however $I_{22} \omega^{2}<\kappa K_{11}$, then replace (6.18), (6.22), and (6.25) by

$$
\begin{aligned}
\tilde{\mathbf{r}}_{1}^{d}= & B_{1}^{\lambda} \cos \left(\alpha_{1} s\right)+B_{2}^{\lambda} \sin \left(\alpha_{1} s\right)+B_{3}^{\lambda} \cosh \left(\alpha_{2} s\right)+B_{4}^{\lambda} \sinh \left(\alpha_{2} s\right), \\
\tilde{\mathbf{a}}_{2}^{d}= & \frac{\omega^{2} \mu-K_{11} \alpha_{1}^{2}}{K_{11} \alpha_{1}}\left(B_{1}^{\lambda} \sin \left(\alpha_{1} s\right)-B_{2}^{\lambda} \cos \left(\alpha_{1} s\right)\right) \\
& +\frac{\omega^{2} \mu+K_{11} \alpha_{2}^{2}}{K_{11} \alpha_{2}}\left(B_{3}^{\lambda} \sinh \left(\alpha_{2} s\right)+B_{4}^{\lambda} \cosh \left(\alpha_{2} s\right)\right),
\end{aligned}
$$

where

$$
\alpha_{2}^{2}=-\omega^{2}\left(\frac{I_{22}}{J_{22}}+\frac{\mu}{K_{11}}\right)+\left(\frac{\omega^{4}}{4}\left(\frac{I_{22}}{J_{22}}-\frac{\mu}{K_{11}}\right)^{2}+\omega^{2} \frac{\kappa \mu}{I_{22}}\right)^{1 / 2} .
$$

If $I_{22} \omega^{2}=\kappa K_{11}$, then replace (6.18) and (6.22) by

$$
\begin{aligned}
& \tilde{\mathbf{r}}_{1}^{d}=B_{1}^{\lambda} \cos \left(\alpha_{1} s\right)+B_{2}^{\lambda} \sin \left(\alpha_{1} s\right)+B_{3}^{\lambda}, \\
& \tilde{\mathbf{a}}_{2}^{d}=\frac{\omega^{2} \mu-K_{11} \alpha_{1}^{2}}{K_{11} \alpha_{1}}\left(B_{1}^{\lambda} \sin \left(\alpha_{1} s\right)-B_{2}^{\lambda} \cos \left(\alpha_{1} s\right)\right)+\frac{B_{3}^{\lambda} \omega^{2} \mu}{K_{11}} s+B_{4}^{\lambda} .
\end{aligned}
$$

If $I_{11} \omega^{2}<\kappa K_{22}$, then replace (6.19), (6.21), and (6.27) by

$$
\begin{aligned}
\tilde{\mathbf{r}}_{2}^{d}= & B_{5}^{\lambda} \cos \left(\alpha_{3} s\right)+B_{6}^{\lambda} \sin \left(\alpha_{3} s\right)+B_{7}^{\lambda} \cosh \left(\alpha_{4} s\right)+B_{8}^{\lambda} \sinh \left(\alpha_{4} s\right), \\
\tilde{\mathbf{a}}_{1}^{d}= & \frac{\omega^{2} \mu-K_{22} \alpha_{3}^{2}}{K_{22} \alpha_{3}}\left(B_{5}^{\lambda} \sin \left(\alpha_{3} s\right)-B_{6}^{\lambda} \cos \left(\alpha_{3} s\right)\right) \\
& +\frac{\omega^{2} \mu+K_{22} \alpha_{4}^{2}}{K_{22} \alpha_{4}}\left(B_{7}^{\lambda} \sinh \left(\alpha_{4} s\right)+B_{8}^{\lambda} \cosh \left(\alpha_{4} s\right)\right),
\end{aligned}
$$


where

$$
\alpha_{4}^{2}=-\omega^{2}\left(\frac{I_{11}}{J_{11}}+\frac{\mu}{K_{22}}\right)+\left(\frac{\omega^{4}}{4}\left(\frac{I_{11}}{J_{11}}-\frac{\mu}{K_{22}}\right)^{2}+\omega^{2} \frac{\kappa \mu}{I_{11}}\right)^{1 / 2} .
$$

If $I_{11} \omega^{2}=\kappa K_{22}$, then replace (6.19) and (6.21) by

$$
\begin{aligned}
& \tilde{\mathbf{r}}_{2}^{d}=B_{5}^{\lambda} \cos \left(\alpha_{3} s\right)+B_{6}^{\lambda} \sin \left(\alpha_{3} s\right)+B_{7}^{\lambda}, \\
& \tilde{\mathbf{a}}_{1}^{d}=\frac{\omega^{2} \mu-K_{22} \alpha_{3}^{2}}{K_{22} \alpha_{3}}\left(B_{5}^{\lambda} \sin \left(\alpha_{3} s\right)-B_{6}^{\lambda} \cos \left(\alpha_{3} s\right)\right)+\frac{B_{7}^{\lambda} \omega^{2} \mu}{K_{22}} s+B_{8}^{\lambda} .
\end{aligned}
$$

It follows that the solution on each line in the net is determined by twelve coefficients $\left\{B_{1}^{\lambda}, \ldots, B_{12}^{\lambda}\right\}$ giving a total of $12 N_{\text {lines }}$ coefficients to be fixed for each mode. Let node $v$ have $N_{\text {lines }}^{v}$ lines attached to it. The continuity of $\underline{\hat{a}}$ at $v$ will give $3\left(N_{\text {lines }}^{v}-1\right)$ linear equations. Equation (4.2) becomes

$$
\tilde{\mathbf{r}}_{\lambda}^{e}(v)=\tilde{\mathbf{r}}^{e}(v)+\tilde{\mathbf{a}}^{e}(v) \times \boldsymbol{\xi}^{d}(v, \lambda)
$$

and writing it in the form

$$
\tilde{\mathbf{r}}_{\lambda_{1}}^{e}(v)-\tilde{\mathbf{r}}_{\lambda_{2}}^{e}(v)=\tilde{\mathbf{a}}^{e}(v) \times\left(\boldsymbol{\zeta}^{d}\left(v, \lambda_{1}\right)-\boldsymbol{\xi}^{d}\left(v, \lambda_{2}\right)\right)
$$

gives rise to a further $3\left(N_{\text {lines }}^{v}-1\right)$ linear equations. The dynamic equations (4.3) and (4.4) become

$$
\begin{gathered}
\omega^{2} M_{\nu}\left(\tilde{\mathbf{r}}_{\lambda_{1}}^{e}-\tilde{\mathbf{a}}^{e} \times \boldsymbol{\xi}^{d}\left(\nu, \lambda_{1}\right)\right)+\sum_{\lambda} \Omega(v, \lambda) \mathbf{K}_{\lambda}^{e}\left(\tilde{\mathbf{r}}_{\lambda}^{e \prime}-\tilde{\mathbf{a}}^{e} \times \mathbf{c}_{\lambda 3}^{e}\right)=0 \\
\omega^{2} \mathbf{I}_{\nu}^{e}\left(\tilde{\mathbf{a}}^{e}\right)+\sum_{\lambda} \Omega(v, \lambda)\left(\mathbf{J}_{\lambda}^{e}\left(\tilde{\mathbf{a}}_{\lambda}^{e \prime}\right)+\kappa \boldsymbol{\xi}^{d}\left(\nu, \lambda_{1}\right) \times \mathbf{K}_{\lambda}^{e}\left(\tilde{\mathbf{r}}_{\lambda}^{e \prime}-\tilde{\mathbf{a}}^{e} \times \mathbf{c}_{\lambda 3}^{e}\right)\right)=0
\end{gathered}
$$

and will give a further two vector equations. Thus each node will specify $6 N_{\text {lines }}^{v}$ equations. It is easy to show that $\sum_{v} N_{\text {lines }}^{v}=2 N_{\text {lines }}$, hence in total there are $12 N_{\text {lines }}$ linear equations in $12 N_{\text {lines }}$ unknowns. These can be written as the matrix equation

$$
M(\omega) \underline{B}=\underline{0},
$$

where $M(\omega)$ is a $12 N_{\text {lines }} \times 12 N_{\text {lines }}$ matrix and $\underline{B}$ is a $12 N_{\text {lines }}$ column vector containing the unknown coefficients. 
Clearly, if $M(\omega)$ is invertible, then $\underline{B}=\underline{0}$. The (eigen) solutions follow from solutions of $\operatorname{det} M(\omega)=0$ with corresponding eigenvectors giving the associated mode shapes.

As stated in the introduction, these equations have been encoded into a Maple computer program LINCOSS [11] which can be used to estimate the mode spectrum and associated eigenmodes of (in principle arbitrary) Cosserat nets containing rigid extended structures.

\section{Rigid bodies as limits of Cosserat elements}

In this section, we show that, by letting the Young and shear moduli $E, G \rightarrow \infty$ of a Kirchhoff-Cosserat element, element becomes a rigid body. Then the element and the two nodes it connects can be replaced by a single extended node, thereby generating a new contracted Cosserat net.

THEOREM 7.1. Let line $\lambda_{c}$ connect the distinct nodes $v_{a}$ and $v_{b}$ in the net $\mathbf{Y}$. Let the Cosserat element represented by the line $\lambda_{c}$ have Kirchhoff constitutive relations, and let $E, G \rightarrow \infty$. If the total energy of any solution of the Cosserat equations is to remain finite in this limit, then this Cosserat element becomes a rigid body. A new contracted net $\tilde{\mathbf{Y}}$ exists, obtained by collapsing the nodes $v_{a}$ and $v_{b}$ and the line $\lambda_{c}$ into a single node $v_{c}$, such that the junction conditions (2.24), (4.2), (4.3), and (4.4) at $\nu_{c}$ are satisfied.

Proof. Let $s_{c}$ be the coordinate running along the line $\lambda_{c}$ which takes values $s^{a} \leq s_{c} \leq s^{b}$. Let the endpoints of line $\lambda_{c}$ be at $v_{a}$ and $v_{b}$ where the orientation points form $v_{a}$ to $v_{b}$.

Configuration space. We construct the new net $\tilde{\mathbf{Y}}$ as follows: the nodes of $\tilde{\mathbf{Y}}$ consist of all the nodes of $\mathbf{Y}$ excluding $\left\{v_{a}, v_{b}\right\}$ and including $v_{c}$. That is, $\mathcal{N}\left(Y^{\prime}\right)=\left\{\boldsymbol{v}_{c}\right\} \cup \mathcal{N}(\mathbf{Y})-\left\{\boldsymbol{v}_{a}, \boldsymbol{v}_{b}\right\}$. The lines of $\tilde{\mathbf{Y}}$ are all the lines in $\mathbf{Y}$ excluding $\lambda_{c}$, that is, $\mathcal{L}\left(Y^{\prime}\right)=\mathcal{L}(\mathbf{Y})-\left\{\lambda_{c}\right\}$. If $v$ is a node in $\mathbf{Y}$ not equal to $v_{a}$ and $v_{b}$, then it has the same connections in $\tilde{\mathbf{Y}}$. If $\lambda$ is connected to $v_{a}$ or $v_{b}$ in $\mathbf{Y}$, then $\lambda$ is connected to $\boldsymbol{v}_{c}$ in $\tilde{\mathbf{Y}}$. See Figure 7.1.

The configuration of the new Cosserat net is encoded into the maps

$$
\begin{array}{ll}
\underline{\tilde{r}}: \mathbb{R} \times \mathbf{Y} \longmapsto \mathbb{R}^{3}, & \tilde{\mathbf{D}}: \mathbb{R} \times \mathbf{Y} \longmapsto \mathrm{SO}(3), \\
\underline{\tilde{r}}:(t, s) \longmapsto \underline{r}(t, s), & \tilde{\mathbf{D}}:(t, s) \longmapsto \tilde{\mathbf{D}}(t, s) .
\end{array}
$$

So we have not yet chosen a reference configuration. If $s$ is on a line other than $\lambda_{c}$ or at a node other than $v_{a}$ or $v_{b}$, then we define

$$
\underline{\tilde{r}}(t, s)=\underline{r}(t, s), \quad \tilde{\mathbf{D}}(t, s)=\mathbf{D}(t, s) \text {. }
$$




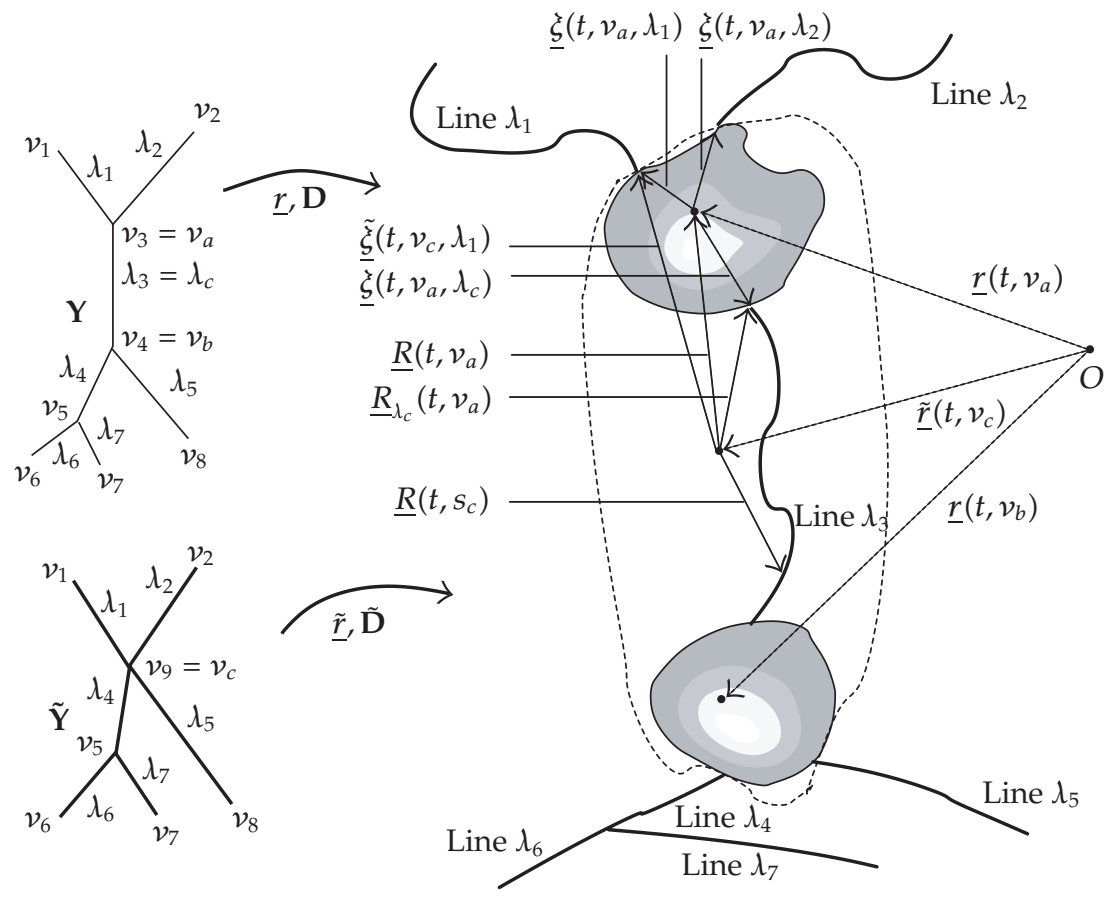

FIgURE 7.1. A Cosserat net with a rigid element at $\lambda_{3}=\lambda_{c}$. Two alternative nets are shown: $Y$ (where the element is considered at the image of the line $\lambda_{3}$ ) and the contacted net $\tilde{\mathbf{Y}}$ (where the element and the rigid bodies at nodes $v_{a}$ and $v_{b}$ are replaced by a single rigid body at $v_{c}$ ). The dashed line represents the domain of an equivalent rigid body at $v_{c}$.

For the new node $v_{c} \in \tilde{\mathbf{Y}}$, then $\underline{\tilde{r}}\left(t, v_{c}\right)$ will be the position of the centroid of the rigid body combining $\bar{v}_{a}, v_{b}$, and the line $\lambda_{c}$. We will be free to choose $\tilde{\mathbf{D}}\left(t, \boldsymbol{v}_{c}\right)$.

Energy considerations. The energy of this Cosserat element is defined by

$$
\begin{aligned}
\varepsilon=\int_{S^{a}}^{s^{b}}\left(\frac{1}{2} \mu \underline{\dot{r}} \cdot \underline{\dot{r}}+\frac{1}{2} \kappa^{-1} \underline{w} \cdot \underline{\mathbf{I}}(\underline{w})+\frac{1}{2}\left(\underline{r}^{\prime}-\underline{d}_{3}\right) \cdot \underline{\mathbf{K}}\left(\underline{r}^{\prime}-\underline{d}_{3}\right)\right. \\
\left.+\frac{1}{2}\left(\underline{u}_{D}-\underline{u}_{0}\right) \cdot \underline{\mathbf{J}}\left(\underline{u}_{D}-\underline{u}_{0}\right)+\varphi(\underline{r}, \tilde{\mathbf{D}})\right) d t d s_{c} .
\end{aligned}
$$

As $E, G \rightarrow \infty$, then the scaling parameters $R_{K}, R_{J} \rightarrow \infty$. It is easy to see, by looking at (3.23), that the diagonal components of $\mathbf{K}^{d}$ and the diagonalized $\mathbf{J}^{d}$, all become infinite. This is true even if $x \rightarrow 0$ since, for example, $\mathbf{K}_{11}^{d}=\chi R_{K}=G A / E_{0} A_{0} \rightarrow \infty$. By looking at the hyperelastic potential 
terms above, in order for $\varepsilon$ to remain bounded, it requires

$$
\underline{u}_{D}-\underline{u}_{0}=0, \quad \underline{r}^{\prime}-\underline{d}_{3}=0
$$

in the limit. The requirement $v_{a} \neq v_{b}$ means that both ends of the Cosserat element not to terminate on the rigid body so there are solutions in which (7.4) hold.

Proof of discontinuity equation for $\tilde{\mathbf{D}}$. Let $\mathbf{C}$ be a reference configuration on $\mathbf{Y}$ which restricted to $\lambda_{c}$ obeys $\mathbf{C}^{\prime}=\mathbf{C} \boldsymbol{\Lambda}\left(\mathbf{u}_{0}^{d}\right)$, with $\mathbf{C}_{\boldsymbol{\lambda}_{c}}\left(\boldsymbol{v}_{a}\right)=\mathbf{X}\left(v_{a}, \lambda_{c}\right)$. From (2.30), it follows that $\mathbf{C}\left(v_{a}\right)=\mathbf{1}_{3}$. From (7.4), we have $\underline{u}_{A}\left(s_{C}\right)=0$ and hence $\mathbf{A}$ is constant along $\lambda_{c}$. Thus write $\mathbf{A}_{c}(t)=\mathbf{A}\left(t, s_{c}\right)$. From the continuity of $\mathbf{A}, \mathbf{A}_{c}(t)=\mathbf{A}\left(t, v_{a}\right)=\mathbf{A}\left(t, v_{b}\right)$. Now, $\mathbf{D}\left(t, v_{a}\right)=\mathbf{A}_{c}(t) \mathbf{C}\left(v_{a}\right)$, so

$$
\mathbf{D}_{\lambda_{c}}\left(t, v_{a}\right)^{T} \mathbf{D}_{\lambda_{c}}\left(t, v_{b}\right)=\mathbf{C}_{\lambda_{c}}\left(v_{a}\right)^{T} \mathbf{C}_{\lambda_{c}}\left(v_{b}\right)=\mathbf{Z},
$$

where we observe that the matrix $\mathbf{Z}$ depends only on the function $\mathbf{u}_{0}^{d}\left(s_{\mathcal{C}}\right)$ that is independent of the choice of $\mathbf{C}$. Thus

$$
\mathbf{D}_{\lambda_{c}}\left(t, v_{b}\right)=\mathbf{D}_{\lambda_{c}}\left(t, v_{a}\right) \mathbf{X}\left(v_{a}, \lambda_{c}\right) \mathbf{Z X}\left(v_{b}, \lambda_{c}\right)^{T}
$$

We choose $\tilde{\mathbf{D}}\left(t, v_{c}\right)=\mathbf{D}\left(t, v_{a}\right)$ then if $\lambda$ is a line connected to $v_{c}$,

$$
\tilde{\mathbf{X}}\left(\lambda, v_{c}\right)= \begin{cases}\mathbf{X}\left(\lambda, v_{a}\right) & \text { if } \lambda \text { is connected to } \boldsymbol{v}_{a} \text { in } \mathbf{Y} \\ \mathbf{X}\left(\lambda_{c}, v_{a}\right) \mathbf{Z X}\left(\lambda_{c}, v_{b}\right)^{T} \mathbf{X}\left(\lambda, v_{b}\right) & \text { if } \lambda \text { is connected to } \boldsymbol{v}_{b} \text { in } \mathbf{Y}\end{cases}
$$

Thus (2.24) follows for the contracted net $\tilde{\mathbf{Y}}$.

Proof of discontinuity equation in $\underline{\tilde{r}}$. Let $\underline{\tilde{r}}\left(t, v_{c}\right)$ be the centroid of the line $\lambda_{c}$ and the masses at the endpoints $v_{a}$ and $v_{b}$. This is given by

$$
\tilde{M}_{v_{c}} \underline{\tilde{r}}\left(t, v_{c}\right)=M_{v_{a}} \underline{r}\left(t, v_{a}\right)+M_{v_{b}} \underline{r}\left(t, v_{b}\right)+\int_{s^{a}}^{s^{b}} \mu\left(s_{c}\right) \underline{r}\left(t, s_{c}\right) d s_{c},
$$

where

$$
\tilde{M}_{v_{c}}=M_{v_{a}}+M_{v_{b}}+\int_{s^{a}}^{s^{b}} \mu\left(s_{c}\right) d s_{c}
$$

is the mass of the new node $v_{c}$. 
We can integrate $\underline{r}^{\prime}=\underline{d}_{3}$ to give

$$
\mathbf{r}_{\lambda_{c}}^{e}\left(t, s_{c}\right)=\mathbf{A}_{c}(t) \int_{s^{a}}^{s_{c}} \mathbf{c}_{3}\left(s_{c}^{\prime}\right) d s_{c}^{\prime}+\mathbf{r}_{\lambda_{c}}^{e}\left(t, s^{a}\right),
$$

where $\mathbf{c}_{3}\left(s_{c}\right)=\mathbf{C}\left(s_{c}\right) \mathbf{e}_{3}$. Then

$$
\begin{aligned}
& \mathbf{r}^{e}\left(t, v_{a}\right)=-\mathbf{A}_{c}(t) \mathbf{C}\left(v_{a}\right) \xi^{d}\left(v_{a}, \lambda_{c}\right)+\mathbf{r}_{\lambda_{c}}^{e}\left(t, s^{a}\right) \\
& \mathbf{r}^{e}\left(t, v_{b}\right)=-\mathbf{A}_{c}(t) \mathbf{C}\left(v_{b}\right) \xi^{d}\left(v_{b}, \lambda_{c}\right)+\int_{s^{a}}^{s^{b}} \mathbf{c}_{3}\left(s_{c}^{\prime}\right) d s_{c}^{\prime}+\mathbf{r}_{\lambda_{c}}^{e}\left(t, s^{a}\right) .
\end{aligned}
$$

Substituting these into (7.8) and dividing by $\tilde{M}_{v_{c}}$ gives

$$
\begin{aligned}
\underline{\underline{r}}\left(t, v_{c}\right)=\mathbf{A}_{c}(t) \frac{1}{\tilde{M}_{v_{c}}}( & -M_{v_{a}} \mathbf{C}\left(v_{a}\right) \boldsymbol{\xi}^{d}\left(v_{a}, \lambda_{c}\right) \\
& +M_{v_{b}}\left(-\boldsymbol{\xi}^{d} \mathbf{C}\left(v_{b}\right)\left(v_{b}, \lambda_{c}\right)+\int_{s^{a}}^{s^{b}} \mathbf{c}_{3}\left(s_{c}^{\prime}\right) d s_{c}^{\prime}\right) \\
& \left.+\int_{s^{a}}^{s^{b}} \mu\left(s_{c}\right) \int_{s^{a}}^{s_{c}} \mathbf{c}_{3}\left(s_{c}^{\prime}\right) d s_{c}^{\prime} d s_{c}\right)+\mathbf{r}_{\lambda_{c}}^{e}\left(t, s^{a}\right) .
\end{aligned}
$$

Substituting into (7.10) gives

$$
\mathbf{r}_{\lambda_{c}}^{e}\left(t, s_{c}\right)=\mathbf{A}_{c}(t) \mathbf{R}_{\lambda_{c}}^{d}\left(s_{c}\right)+\tilde{\mathbf{r}}^{e}\left(t, v_{c}\right),
$$

where

$$
\begin{aligned}
\mathbf{R}_{\lambda_{c}}^{d}\left(s_{c}\right)=\int_{s^{a}}^{s_{c}} \mathbf{c}_{3}\left(s_{c}^{\prime}\right) d s_{c}^{\prime} & \\
+\frac{1}{\tilde{M}_{v_{c}}}( & -M_{v_{a}} \mathbf{C}\left(\nu_{a}\right) \boldsymbol{\xi}^{d}\left(\nu_{a}, \lambda_{c}\right) \\
& +M_{v_{b}}\left(-\mathbf{C}\left(\nu_{b}\right) \xi^{d}\left(\nu_{b}, \lambda_{c}\right)+\int_{s^{a}}^{s^{b}} \mathbf{c}_{3}\left(s_{c}^{\prime}\right) d s_{c}^{\prime}\right) \\
& \left.+\int_{s^{a}}^{s^{b}} \mu\left(s_{c}^{\prime}\right) \int_{s^{a}}^{s_{c}^{\prime}} \mathbf{c}_{3}\left(s_{c}^{\prime \prime}\right) d s_{c}^{\prime \prime} d s_{c}^{\prime}\right) .
\end{aligned}
$$

Since $\mathbf{R}_{\lambda_{c}}^{d}\left(s_{c}\right)$ is independent of $t$, it defines a point on a rigid body with body frame $\left\{\underline{d}_{j}\right\}$. Writing $\mathbf{R}_{\lambda_{c}}^{d}(s)=\mathbf{R}_{\lambda_{c}}^{d}\left(s_{c}\right)$, we extend the definition of $\mathbf{R}^{d}$ to a function on the subnet of $\mathbf{Y}$ given by $v_{a}, v_{b}$, and $\lambda_{c}$, where $s_{c}$ is the 
coordinate of the point $s$, so that $\mathbf{R}_{\lambda_{c}}^{d}\left(\mathcal{V}_{a}\right)=\mathbf{R}_{\lambda_{c}}^{d}\left(s^{a}\right)$ and $\mathbf{R}_{\lambda_{c}}^{d}\left(\mathcal{v}_{b}\right)=\mathbf{R}_{\lambda_{c}}^{d}\left(s^{b}\right)$. Further, define

$$
\mathbf{R}^{d}(s)= \begin{cases}\mathbf{R}_{\lambda_{c}}^{d}(s) & \text { if } s \text { is an interior point in } \lambda_{c} \\ \mathbf{R}_{\lambda_{c}}^{d}\left(v_{a}\right)-\mathbf{C}\left(v_{a}\right) \xi^{d}\left(v_{a}, \lambda_{c}\right) & \text { if } s=v_{a}, \\ \mathbf{R}_{\lambda_{c}}^{d}\left(v_{b}\right)-\mathbf{C}\left(v_{b}\right) \xi^{d}\left(v_{b}, \lambda_{c}\right) & \text { if } s=v_{b}\end{cases}
$$

so $\mathbf{R}^{d}\left(v_{a}\right)$ is the centroid of the rigid body at $v_{a}$ with respect to the centroid of the combined body. As before, define the column vector and vector

$$
\mathbf{R}^{e}(t, s)=\mathbf{A}_{c}(t) \mathbf{R}^{d}(s), \quad \underline{R}(t, s)=\mathbf{R}^{e}(t, s) \cdot \mathbf{e}_{j} \underline{e}_{j} .
$$

Thus $\underline{\tilde{r}}$ obeys the discontinuity condition (4.2) at $v_{c}$ with

$$
\tilde{\boldsymbol{\xi}}^{d}\left(v_{c}, \lambda\right)= \begin{cases}\mathbf{R}^{d}\left(v_{a}\right)+\xi^{d}\left(v_{a}, \lambda\right) & \text { if } \lambda \text { is connected to } v_{a} \text { in } \mathbf{Y} \\ \mathbf{R}^{d}\left(v_{b}\right)+\xi^{d}\left(v_{b}, \lambda\right) & \text { if } \lambda \text { is connected to } v_{b} \text { in } \mathbf{Y}\end{cases}
$$

Proof of $\ddot{\tilde{r}}$ equation. For the rest of this proof, we drop the explicit $t$ in all arguments. Although the entries in $\mathbf{K}^{d}$ and $\mathbf{J}^{d}$ are infinite, the limits $\mathbf{n}^{d}=$ $\mathbf{K}^{d}\left(\mathbf{v}^{d}-\mathbf{e}_{3}\right)$ and $\mathbf{m}^{d}=\mathbf{J}^{d}\left(\mathbf{u}_{D}^{d}-\underline{u}_{0}\right)$ are well defined and not necessarily zero. Differentiating (7.8) twice with respect to $t$ gives

$$
\begin{aligned}
\int_{s^{a}}^{s^{b}} \mu \ddot{r} d s_{c} & =\tilde{M}_{v_{c}} \ddot{\tilde{r}}\left(v_{c}\right)-M_{v_{a}} \ddot{\ddot{r}}\left(v_{a}\right)-M_{v_{b}} \ddot{\ddot{r}}\left(v_{b}\right) \\
& =\int_{s^{a}}^{s^{b}}\left(\underline{n}^{\prime}+\underline{f}\left(t, s_{c}\right)\right) d s_{c} \\
& =\underline{n}_{\lambda_{c}}\left(v_{b}\right)-\underline{n}_{\lambda_{c}}\left(v_{a}\right)+\underline{\tilde{F}}_{v_{c}}-\underline{F}_{v_{a}}-\underline{F}_{v_{b}}
\end{aligned}
$$

where $\underline{\tilde{F}}_{v_{c}}(t)=\int \underline{f}\left(t, s_{c}\right) d s_{c}+\underline{F}_{v_{a}}+\underline{F}_{v_{b}}$ is the total force along the element and at its endpoints. The junction equations for $\underline{\ddot{r}}$ (4.3) may be written as

$$
\begin{aligned}
& M_{v_{a}} \ddot{\ddot{r}}\left(v_{a}\right)=\underline{F}_{v_{a}}-\sum_{\lambda \neq \lambda_{c}} \Omega\left(v_{a}, \lambda\right) \underline{n}_{\lambda}\left(t, v_{a}\right)+\underline{n}_{\lambda_{c}}\left(t, v_{a}\right), \\
& M_{v_{b}} \ddot{\ddot{r}}\left(v_{b}\right)=\underline{F}_{v_{b}}-\sum_{\lambda \neq \lambda_{c}} \Omega\left(v_{b}, \lambda\right) \underline{n}_{\lambda}\left(t, v_{b}\right)-\underline{n}_{\lambda_{c}}\left(t, v_{b}\right) .
\end{aligned}
$$


Adding these together gives

$$
\tilde{M}_{v_{c}} \underline{\ddot{r}}\left(v_{c}\right)=\underline{\tilde{F}}_{v_{c}}-\sum_{\lambda \neq \lambda_{c}} \Omega\left(v_{a}, \lambda\right) \underline{n}_{\lambda}\left(t, v_{a}\right)-\sum_{\lambda \neq \lambda_{c}} \Omega\left(v_{b}, \lambda\right) \underline{n}_{\lambda}\left(t, v_{b}\right),
$$

the extended rigid node equation (4.3) for node $v_{c}$ in the configuration space $\tilde{\mathbf{Y}}$.

Proof of $\tilde{\mathbf{I}}_{v_{c}}(\underline{\tilde{w}})^{\cdot}$ equation. Let $\underline{\mathbf{I}}_{v_{c}}$ be the total moment of inertia of the element about its centroid, given in the director frame by

$$
\begin{aligned}
\tilde{\mathbf{I}}_{v_{c}}^{d}= & \int_{s^{a}}^{s^{b}}\left(\mathbf{I}^{d}\left(s_{c}\right)+\kappa \mu\left(s_{c}\right) \mathbf{M}\left(\mathbf{R}^{d}\left(s_{c}\right)\right)\right) d s_{c} \\
& +\mathbf{I}_{v_{a}}^{d}+\kappa M_{v_{a}} \mathbf{M}\left(\mathbf{R}^{d}\left(v_{a}\right)\right)+\mathbf{I}_{v_{b}}^{d}+\kappa M_{v_{b}} \mathbf{M}\left(\mathbf{R}^{d}\left(v_{b}\right)\right),
\end{aligned}
$$

where $\mathbf{M}(\mathbf{x})$ is the matrix $\mathbf{M}(\mathbf{x})=\|\mathbf{x}\| \mathbf{1}_{3}-\mathbf{x} \mathbf{x}^{T}$ so that $\mathbf{M}(\mathbf{x}) \mathbf{y}=\mathbf{x} \times(\mathbf{y} \times \mathbf{x})$. Similarly, define the $\left(\begin{array}{l}1 \\ 1\end{array}\right)$ tensor $\underline{\mathbf{M}}(\underline{x})$. In order to integrate (3.3), we need the following:

$$
\begin{aligned}
\int_{s^{a}}^{s^{b}} \underline{r}^{\prime} \times \underline{n} d s_{c}= & \int_{s^{a}}^{s^{b}} \underline{R^{\prime}} \times \underline{n} d s_{c}=[\underline{R} \times \underline{n}]_{S^{a}}^{s^{b}}-\int_{s^{a}}^{s^{b}} \underline{R} \times \underline{n}^{\prime} d s_{C} \\
= & \underline{R}_{\lambda_{c}}\left(\mathcal{v}_{b}\right) \times \underline{n}_{\lambda_{c}}\left(\mathcal{v}_{b}\right)-\underline{R}_{\lambda_{c}}\left(\mathcal{v}_{a}\right) \\
& \times \underline{n}_{\lambda_{c}}\left(v_{a}\right)-\int_{S^{a}}^{s^{b}} \underline{R} \times(\mu \underline{\ddot{r}}-\underline{f}) d s_{c} .
\end{aligned}
$$

In the last term, $\int_{s^{a}}^{s^{b}} \underline{R} \times \underline{f} d s_{c}$ contributes to the overall torque $\underline{\tilde{L}}_{v_{c}}$. Whilst

$$
\begin{aligned}
-\int_{s^{a}}^{s^{b}} \mu \underline{R}\left(s_{c}\right) \times \underline{\ddot{r}}\left(s_{c}\right) d s_{c} \\
\quad=-\int_{s^{a}}^{s^{b}} \mu \underline{R}\left(s_{c}\right) \times \underline{\ddot{R}}\left(s_{c}\right) d s_{c}-\int_{s^{a}}^{s^{b}} \mu \underline{R}\left(s_{c}\right) \times \underline{\ddot{r}}\left(v_{c}\right) d s_{c} \\
=-\int_{s^{a}}^{s^{b}} \mu\left(\underline{\mathbf{M}}\left(\underline{R}\left(s_{c}\right)\right) \underline{\tilde{\tilde{w}}}\right)^{\cdot} d s_{c}-\left(\int_{s^{a}}^{s^{b}} \mu \underline{R}\left(s_{c}\right) d s_{c}\right) \times \underline{\ddot{\tilde{r}}}\left(\mathcal{v}_{c}\right),
\end{aligned}
$$


since $\underline{R} \times \underline{\ddot{R}}=(\underline{R} \times \underline{\dot{R}})^{\cdot}=(\underline{R} \times(\underline{\tilde{w}} \times \underline{R}))^{\cdot}=(\underline{\mathbf{M}}(\underline{R}) \underline{\tilde{w}})^{\cdot}$ and

$$
\dot{\mathbf{R}}^{e}(t, s)=\dot{\mathbf{A}}_{c}(t) \mathbf{R}^{d}(s)=\Lambda\left(\tilde{\mathbf{w}}^{e}(t)\right) \mathbf{A}_{c}(t) \mathbf{R}^{d}(s)=\tilde{\mathbf{w}}^{e}(t) \times \mathbf{R}^{e}(t, s) .
$$

Now,

$$
\begin{aligned}
&-\left(\int_{s^{a}}^{s^{b}} \mu \underline{R}\left(s_{c}\right) d s_{c}\right) \times \underline{\tilde{r}}\left(v_{c}\right) \\
&=\left(M_{v_{a}} \underline{R}\left(v_{a}\right)+M_{v_{b}} \underline{R}\left(v_{b}\right)\right) \times \underline{\ddot{r}}\left(v_{c}\right) \\
&= M_{v_{a}} \underline{R}\left(v_{a}\right) \times \underline{\tilde{r}}\left(v_{a}\right)-M_{v_{a}} \underline{R}\left(v_{a}\right) \times \underline{\ddot{R}}\left(v_{a}\right) \\
&+M_{v_{b}} \underline{R}\left(v_{b}\right) \times \underline{\ddot{r}}\left(v_{b}\right)-M_{v_{b}} \underline{R}\left(v_{b}\right) \times \underline{\ddot{R}}\left(v_{b}\right) \\
&=-M_{v_{a}}\left(\underline{\mathbf{M}}\left(\underline{R}\left(v_{a}\right)\right) \underline{\tilde{w}}\right)-M_{v_{b}}\left(\underline{\mathbf{M}}\left(\underline{R}\left(v_{b}\right)\right) \underline{\tilde{w}}\right) \\
&+\underline{R}\left(v_{a}\right) \times \underline{F}\left(v_{a}\right)-\underline{R}\left(v_{a}\right) \times \sum_{\lambda} \Omega\left(v_{a}, \lambda\right) \underline{n}_{\lambda}\left(t, v_{a}\right) \\
&+\underline{R}\left(v_{b}\right) \times \underline{F}\left(v_{b}\right)-\underline{R}\left(v_{b}\right) \times \sum_{\lambda} \Omega\left(v_{b}, \lambda\right) \underline{n}_{\lambda}\left(t, v_{b}\right) .
\end{aligned}
$$

Hence,

$$
\begin{aligned}
\int_{s^{a}}^{s^{b}} & \underline{r}^{\prime} \times \underline{n} d s_{c} \\
= & -\int_{s^{a}}^{s^{b}} \mu(\underline{\mathbf{M}}(\underline{R}) \underline{\tilde{w}})^{\cdot} d s_{c}-M_{v_{a}}\left(\underline{\mathbf{M}}\left(\underline{R}\left(v_{a}\right)\right) \underline{\tilde{w}}\right)^{\cdot}-M_{v_{b}}\left(\underline{\mathbf{M}}\left(\underline{R}\left(v_{b}\right)\right) \underline{\tilde{w}}\right)^{.} \\
& +\int_{s^{a}}^{s^{b}} \underline{R} \times \underline{f} d s_{c}+\underline{R}\left(v_{a}\right) \times \underline{F}\left(v_{a}\right)+\underline{R}\left(v_{b}\right) \times \underline{F}\left(v_{b}\right) \\
& +\underline{R}\left(v_{a}\right) \times \sum_{\lambda \neq \lambda_{c}} \Omega\left(v_{a}, \lambda\right) \underline{n}_{\lambda}\left(t, v_{a}\right)+\underline{R}\left(v_{b}\right) \times \sum_{\lambda \neq \lambda_{c}} \Omega\left(v_{b}, \lambda\right) \underline{n}_{\lambda}\left(t, v_{b}\right) .
\end{aligned}
$$

Integrating (3.3) along the element gives

$$
\begin{aligned}
\left(\int_{s^{a}}^{s^{b}} \underline{\mathbf{I}}\left(s_{c}\right) \underline{\tilde{w}} d s_{c}\right) & =\int_{s^{a}}^{s^{b}}\left(\underline{m}^{\prime}+\kappa \underline{r}^{\prime} \times \underline{n}+\underline{l}\right) d s_{c} \\
& =\underline{m}_{\lambda_{c}}\left(v_{b}\right)-\underline{m}_{\lambda_{c}}\left(v_{a}\right)+\kappa \int_{s^{a}}^{s^{b}} \underline{r}^{\prime} \times \underline{n} d s_{c}+\int_{s^{a}}^{s^{b}} l d s_{c} .
\end{aligned}
$$


The two junction conditions (4.4) may be written as

$$
\begin{aligned}
& \left(\underline{\mathbf{I}}_{v_{a}} \underline{\tilde{w}}\right)^{\cdot}=\underline{L}\left(v_{a}\right)-\sum_{\lambda \neq \lambda_{c}} \Omega\left(v_{a}, \lambda\right)\left(\underline{m}_{\lambda}\left(v_{a}\right)+\kappa \underline{\xi}^{\xi}\left(v_{a}, \lambda\right) \times \underline{n}_{\lambda}\left(v_{a}\right)\right) \\
& +\underline{m}_{\lambda_{c}}\left(v_{a}\right)+\kappa \underline{\xi}\left(v_{a}, \lambda_{c}\right) \times \underline{n}_{\lambda_{c}}\left(v_{a}\right), \\
& \left(\underline{\mathbf{I}}_{v_{a}} \underline{\tilde{w}}\right)^{\cdot}=\underline{L}\left(v_{b}\right)-\sum_{\lambda \neq \lambda_{c}} \Omega\left(v_{b}, \lambda\right)\left(\underline{m}_{\lambda}\left(v_{b}\right)+\kappa \underline{\xi}\left(v_{b}, \lambda\right) \times \underline{n}_{\lambda}\left(v_{b}\right)\right) \\
& -\underline{m}_{\lambda_{c}}\left(v_{b}\right)-\kappa \underline{\xi}\left(v_{b}, \lambda_{c}\right) \times \underline{n}_{\lambda_{c}}\left(v_{b}\right) .
\end{aligned}
$$

Adding all three equations together gives

$$
\begin{aligned}
\left(\underline{\tilde{I}}_{v_{c}} \tilde{\tilde{w}}\right)^{\cdot}= & \underline{L}\left(v_{a}\right)+\underline{L}\left(v_{b}\right)+\int_{s^{a}}^{s^{b}} l d s_{c} \\
& +\kappa \int_{s^{a}}^{s^{b}} \underline{R} \times \underline{f} d s_{c}+\kappa \underline{R}\left(v_{a}\right) \times \underline{F}\left(v_{a}\right)+\kappa \underline{R}\left(v_{b}\right) \times \underline{F}\left(v_{b}\right) \\
& +\sum_{\lambda \neq \lambda_{c}} \Omega\left(v_{a}, \lambda\right) \underline{m}_{\lambda}\left(v_{a}\right)+\sum_{\lambda \neq \lambda_{c}} \Omega\left(v_{b}, \lambda\right) \underline{m}_{\lambda}\left(v_{b}\right) \\
& +\kappa \sum_{\lambda \neq \lambda_{c}} \Omega\left(v_{a}, \lambda\right) \underline{\xi}\left(v_{a}, \lambda\right) \times \underline{n}_{\lambda}\left(v_{a}\right) \\
& +\kappa \underline{R}\left(v_{a}\right) \times \sum_{\lambda \neq \lambda_{c}} \Omega\left(v_{a}, \lambda\right) \underline{n}_{\lambda}\left(t, v_{a}\right) \\
& +\kappa \sum_{\lambda \neq \lambda_{c}} \Omega\left(v_{b}, \lambda\right) \underline{\xi}\left(v_{b}, \lambda\right) \times \underline{n}_{\lambda}\left(v_{b}\right) \\
& +\kappa \underline{R}\left(v_{b}\right) \times \sum_{\lambda \neq \lambda_{c}} \Omega\left(v_{b}, \lambda\right) \underline{n}_{\lambda}\left(t, v_{b}\right) \\
= & \underline{\tilde{L}}_{v_{c}}+\sum_{\lambda \neq \lambda_{c}} \Omega\left(v_{a}, \lambda\right) \underline{m}_{\lambda}\left(v_{a}\right)+\sum_{\lambda \neq \lambda_{c}} \Omega\left(v_{b}, \lambda\right) \underline{m}_{\lambda}\left(v_{b}\right) \\
& +\kappa \sum_{\lambda \neq \lambda_{c}} \Omega\left(v_{a}, \lambda\right) \underline{\tilde{\xi}}\left(v_{c}, \lambda\right) \times \underline{n}_{\lambda}\left(v_{a}\right) \\
& +\kappa \sum_{\lambda \neq \lambda_{c}} \Omega\left(v_{b}, \lambda\right) \underline{\tilde{\xi}}\left(v_{b}, \lambda\right) \times \underline{n}_{\lambda}\left(v_{b}\right)
\end{aligned}
$$

where $\underline{\tilde{v}}_{v_{c}}$ is the total torque about the centroid. Hence, (4.4) is satisfied for $v_{c}$ in $\tilde{\mathbf{Y}}$. 


\section{Conclusion and discussion}

In this paper we have formulated the dynamics of a collection of connected simple 1-dimensional Cosserat structures and rigid bodies in terms of sections of an $\mathrm{SO}(3)$ fibration over a 1-dimensional net. The junction conditions considered have been restricted to maintain certain rigidity constraints throughout the dynamic evolution of the Cosserat net. The equations of motion and junction conditions have been derived as extrema of a constrained variational principle on the net. The existence of such an action makes possible approximation schemes for the determination of the dynamic history of the net given initial conditions, constitutive data for the members of the net, and the applied external forces and torques. For perturbations about a stress-free configuration of a Kirchhoff-Cosserat net, we have outlined a normal mode analysis for such a history. Limits have been discussed that effect transformations of Cosserat elements to rigid bodies thereby contracting the Cosserat net.

Although the class of junction considered explicitly here is large, there is a host of generalisations that can be accommodated within this framework. One may relax certain of the rigidity conditions at various junctions and consider frictional forces and torques where sliding is permitted. Thus one might consider junctions with a universal joint with or without friction or restoring torques. These could be analysed by allowing $\mathbf{A}$ to be discontinuous at such nodes with restoring torques dependent of $\mathbf{A}_{\mathcal{\lambda}}(t, v) \mathbf{A}(t, v)^{T}$. (In this paper, this is the identity matrix at each node corresponding to the prohibition of angular motion at the junction.) Alternatively, constrained rotary motion at a junction can be considered by restricting the angular freedom of the direction of contact, that is, $\mathbf{A}_{\lambda}(t, v) \mathbf{A}(t, v)^{T}$ is restricted to some subset of $\mathrm{SO}(3)$.

Another generalisation would be to consider the dynamics of nets with mobile nodes. Examples include the motion of a ring on a wire, a wire sliding over a fixed or moving point in space, or two wires in intermittent contact. These and other applications of the Cosserat net description of connected slender structures will be discussed elsewhere.

\section{References}

[1] F. J. Almgren Jr., Plateau's Problem: An Invitation to Varifold Geometry, W. A. Benjamin, New York, 1966.

[2] S.S. Antman, Nonlinear Problems of Elasticity, Applied Mathematical Sciences, vol. 107, Springer-Verlag, New York, 1995.

[3] R. Beccu, C. M. Wu, and B. Lundberg, Reflection and transmission of the energy of transient elastic extensional waves in a bent bar, J. Sound Vibration 191 (1996), no. 2, 261-272.

[4] A. Bermudez and J. M. Viaño, Une justification des équations de la thermoélasticité des poutres à section variable par des méthodes asymptotiques [A justification 
of thermoelastic equations for variable-section beams by asymptotic methods], RAIRO Anal. Numér. 18 (1984), no. 4, 347-376 (French).

[5] D. Q. Cao, R. W. Tucker, and C. H. T. Wang, Cosserat aeroelastic stability of cable-stayed bridges, Proceedings of the 4th International Symposium on Cable Dynamics, Montreal, Canada, 2001.

[6] P. G. Ciarlet, H. Le Dret, and R. Nzengwa, Junctions between three-dimensional and two-dimensional linearly elastic structures, J. Math. Pures Appl. (9) 68 (1989), no. 3, 261-295.

[7] A. Cimetière, G. Geymonat, H. Le Dret, A. Raoult, and Z. Tutek, Asymptotic theory and analysis for displacements and stress distribution in nonlinear elastic straight slender rods, J. Elasticity 19 (1988), no. 2, 111-161.

[8] P. Desmond, Theoretical and experimental investigation of stress waves at a junction, J. Appl. Mech. 48 (1981), 148-154.

[9] J. F. Doyle and S. Kamle, An experimental study of the reflection and transmission of flexural at an arbitrary T-joint, J. Appl. Mech. 54 (1987), 136-140.

[10] S. Gaudet, C. Gauthier, and V. G. LeBlanc, On the vibrations of an N-string, J. Sound Vibration 238 (2000), no. 1, 147-169.

[11] J. G. Gratus and R. W. Tucker, LINCOSS, a Maple program for the mode analysis of Cosserat nets, Lancaster University, Lancaster, 2002.

[12] H. Le Dret, Modeling of the junction between two rods, J. Math. Pures Appl. (9) 68 (1989), no. 3, 365-397.

[13] J. P. Lee and H. Kolsky, The generation of stress pulses at the junction of two noncollinear rods, J. Appl. Math. Mech. 39 (1972), 809-813.

[14] E. J. P. G. Schmidt, On the modelling and exact controllability of networks of vibrating strings, SIAM J. Control Optim. 30 (1992), no. 1, 229-245.

[15] J. C. Simo, J. E. Marsden, and P. S. Krishnaprasad, The Hamiltonian structure of nonlinear elasticity: the material and convective representations of solids, rods, and plates, Arch. Rational Mech. Anal. 104 (1988), no. 2, 125-183.

[16] J. C. Simo, T. A. Posbergh, and J. E. Marsden, Stability of coupled rigid body and geometrically exact rods: block diagonalization and the energy-momentum method, Phys. Rep. 193 (1990), no. 6, 279-360.

[17] R. W. Tucker and C. H. T. Wang, An integrated model for drill-string dynamics, J. Sound Vibration 224 (1999), no. 1, 123-165.

[18] Z. Tutek and I. Aganović, A justification of the one-dimensional linear model of elastic beam, Math. Methods Appl. Sci. 8 (1986), no. 4, 502-515.

[19] P. C. Varadi, Conditions for stability of rotating elastic rods, R. Soc. Lond. Proc. Ser. A Math. Phys. Eng. Sci. 457 (2001), no. 2011, 1701-1720.

[20] C. M. Wu and B. Lundberg, Reflection and transmission of the energy of harmonic elastic waves in a bent bar, J. Sound Vibration 190 (1996), no. 4, 645-659.

[21] K. H. Yong and K. J. Atkins, Generation of elastic stress waves at a T-junction of square rods, J. Sound Vibration 88 (1983), no. 4, 431-436.

J. Gratus and R. W. Tucker: Department of Physics, Lancaster University, Bailrigg, Lancaster, LA 4YB, UK 


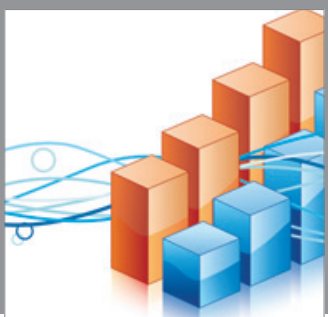

Advances in

Operations Research

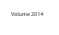

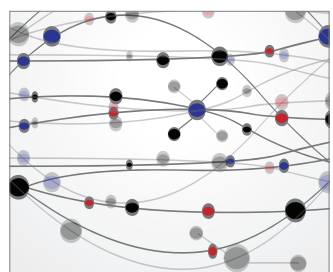

\section{The Scientific} World Journal
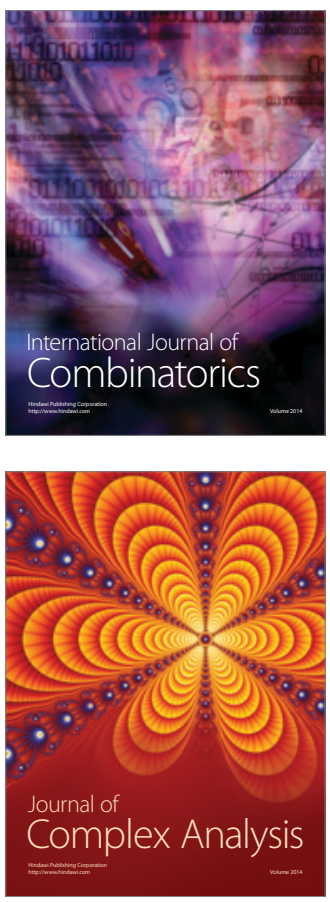

International Journal of

Mathematics and

Mathematical

Sciences
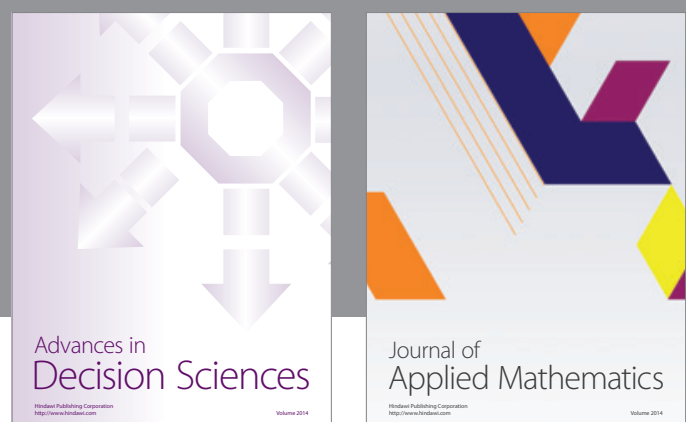

Journal of

Applied Mathematics
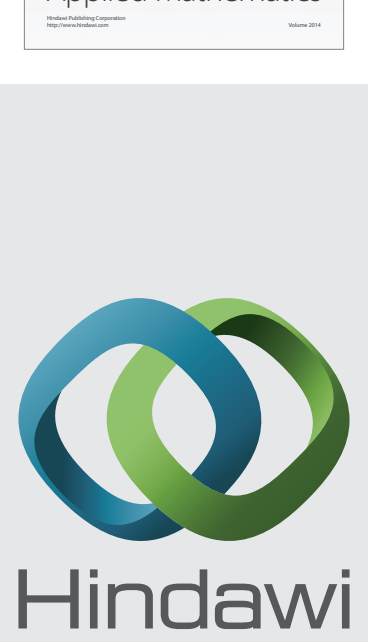

Submit your manuscripts at http://www.hindawi.com
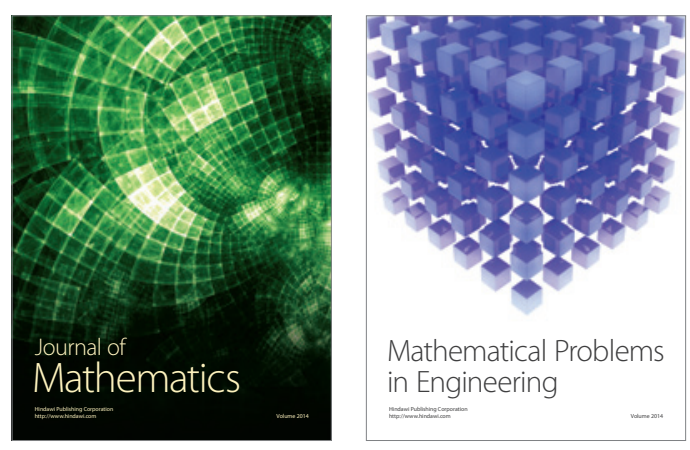

Mathematical Problems in Engineering
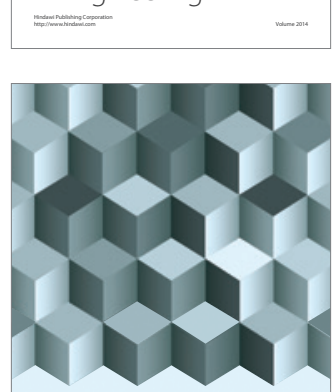

Journal of

Function Spaces
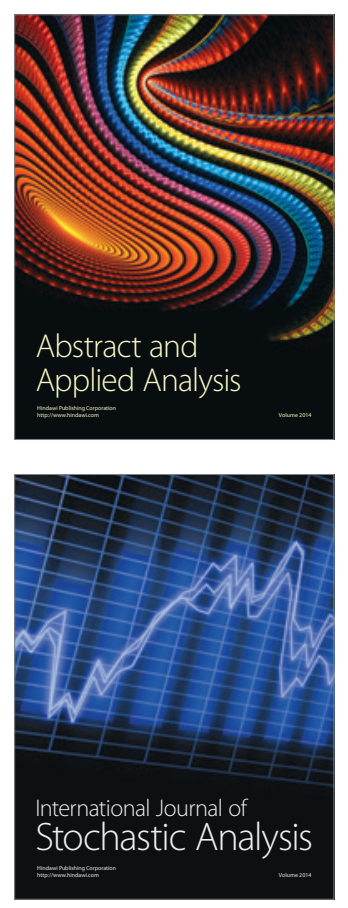

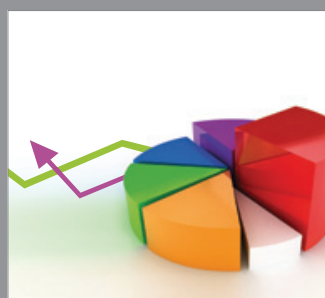

ournal of

Probability and Statistics

Promensencen
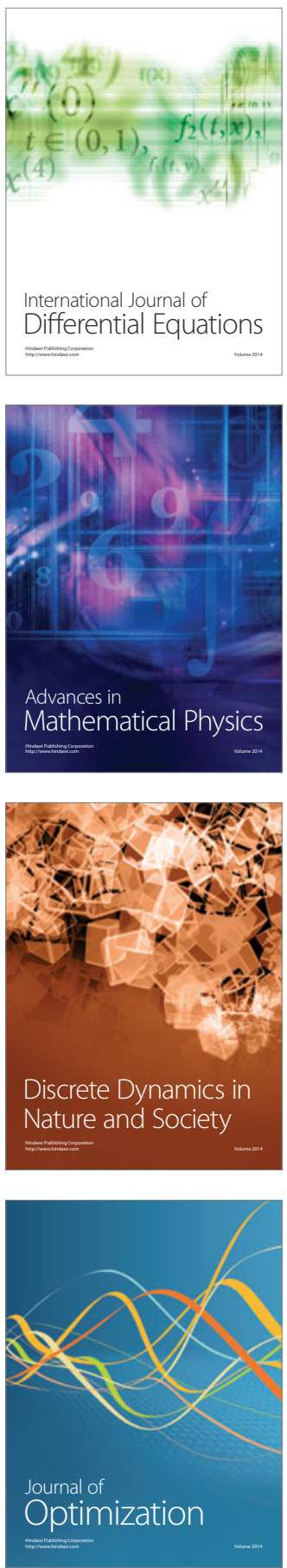